\title{
Synthesis and cytotoxic properties of alkynic triterpenoid Mannich compounds
}

\author{
Ronny Sczepek ${ }^{1}$, Christoph Nitsche ${ }^{1}$, Lucie Heller ${ }^{1}$, Bianka Siewert ${ }^{1}$, Renate Schäfer ${ }^{1}$, Franziska \\ Flemming ${ }^{1}$, Choijiljav Otgonbayar ${ }^{2}$ and René Csuk ${ }^{1, *}$ \\ ${ }^{1}$ Martin-Luther Universität Halle-Wittenberg, Organische Chemie, Kurt-Mothes- Str. 2, D-06120 Halle (Saale), \\ Germany \\ ${ }^{2}$ Institute of Chemistry and Chemical Technology, Mongolian Academy of Sciences, Ulaanbaatar-51, Mongolia
}

\begin{abstract}
In summary, we prepared alkynic Mannich bases of betulinic or glycyrrhetinic acid; many of these compounds showed an improved cytotoxicity as compared to their parent compounds glycyrrhetinic or betulinic acid. The highest cytotoxicity was determined for a Mannich compound derived from glycyrrhetinic acid, and $\mathrm{EC}_{50}$ values as low as $3.3 \mu \mathrm{M}$ were achieved for this compound employing the human submandibular carcinoma cell line A253. As proven by AO/PI staining as well as by DNA-laddering experiments this compound acts by apoptosis.
\end{abstract}

Keywords: Betulin; Betulinic acid; Glycyrrhetinic acid; Mannich compounds; Antitumor.

\section{Introduction}

The therapy of cancer is still a very important medicinal issue. Although there has been an enormous progress in the treatment of cancer, improvements in therapy, prophylaxis and the

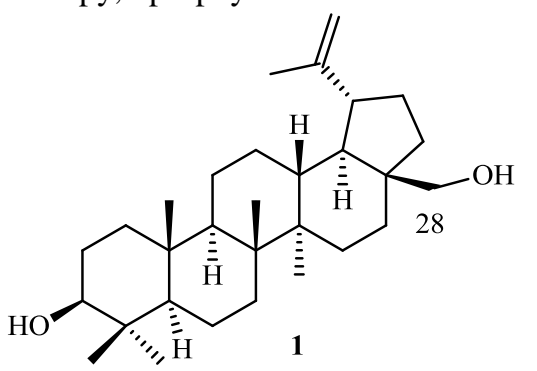

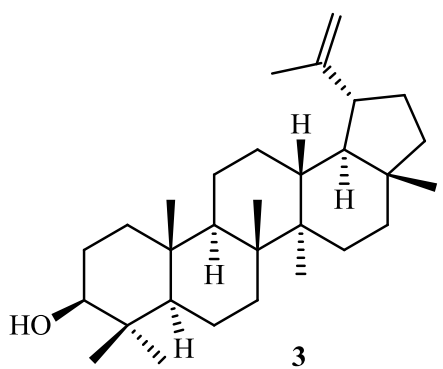

development of more selective drugs are still called for and remain a critical topic. Recently, derivatives of betulin 1 and betulinic acid 2 (Fig. 1) have quite successfully passed in-vitro tests, and preliminary preclinical in-vivo trials gave good results ${ }^{1,2}$.<smiles>CCCC(C)(C)CO</smiles>

Figure 1. Structure of triterpenoids betulin 1, betulinic acid 2, lupeol 3 and glycyrrhetinic acid 4.

Thus, several of these candidates were selected for an extended preclinical testing and clinical

* Corresponding author: René Csuk

E-mail address: rene.csuk@chemie.uni-halle.de

DOI: http://dx.doi.org/10.13171/mjc.4.3.2015.05.26.14.18/csuk investigations are expected to start soon ${ }^{1}$. Triterpenoic compounds of the betulin-type are 
usually synthesized from their parent compounds $\mathbf{1}$ and $\mathbf{2}$, respectively. Betulin $\mathbf{1}$ and to a lesser extent betulinic acid 2 can be extracted from various plant materials $^{3,4}$. The isolation of $\mathbf{1}$ from renewable resources seems of special interest. Hence, there are numerous reports and patents on the extraction of $\mathbf{1}$ from birch bark. The latter is a cheap by-product in wood processing. Several kinds of birch trees have been investigated so far for their content of $\mathbf{1}$, and Betula platyphylla seems a very promising tree. It can grow very easily, and is abundant in many areas of Eurasia. This makes this tree a very interesting source for the isolation of natural products. There are several reports on improving the yield ${ }^{5}$ of $\mathbf{1}$ or manipulating $^{6}$ the tree during its growing. Unfortunately, less work has been accomplished to investigate how to extract $\mathbf{1}$ in an economic, efficient and high yielding manner.

\section{Results and Discussion}

As far as cytotoxic betulin derivatives are concerned, two major problems, are to be investigated in more detail: Firstly, we searched for and tried to develop drugs being more selective for cancer cells than to nonmalignant cells, secondly we wanted to develop their economic synthesis. Usually for the extraction of betulin, the plant material is dried, crunched and extracted with solvents like toluene $^{7}$, several water-organic-solvent mixtures ${ }^{8}$, supercritical $\mathrm{CO}_{2}{ }^{9,}{ }^{10}$ or ionic liquids ${ }^{11}$. Given the physico-chemical properties of $1(\log \mathrm{P}=9.01 \pm$ $0.39)^{12}$, a comparison between the extraction with hexanes (according to literature) and diethyl ether was performed. While the extraction of finely powdered dry birch bark with hexanes for $15 \mathrm{~h}$ in a Soxhlet apparatus furnished only $10.5 \%$ (recovery from the weight of bark submitted to extraction) of an extract containing $47 \%$ betulin and $43 \%$ of lupeol, the extraction with diethyl ether under the same conditions yielded $27.2 \%$ recovery. This extract consisted of $5 \%$ lupeol and $81 \%$ of betulin that could easily be separated by chromatography. Thus, the extraction of the birch bark with ether is more efficient and rewarding than its extraction with hexane.

Recently we were able to show that alkynic Mannich bases derived from triterpenoids showed promising cytotoxic activity ${ }^{13,14}$. In this study, we are interested in a comparison of analogous derivatives of betulin, betulinic acid as well as of glycyrrhetinic acid 4.

Jones oxidation of $\mathbf{1}$ furnished betulonic acid $\mathbf{5}$ (Scheme 1$)^{15,16}$, whose reaction with oxalyl chloride gave chloride 6. Reaction of compound 6 with propargylic alcohol yielded alkyne 7 (70\% yield). This compound is characterized in its ${ }^{13} \mathrm{C}$ NMR spectrum by the presence of signals at $\delta=78.1$ and $74.3 \mathrm{ppm}$ being assigned to the alkyne moiety. Mannich reaction of $\mathbf{7}$ with diisopropylamine and formalin in the presence of $\mathrm{CuI}^{17}$ furnished $\mathbf{8}$. In an analogous manner, from the reaction of $\mathbf{7}^{18,19}$ with diallylamine, morpholine, thiomorpholine or benzylamine the products $\mathbf{9 - 1 2}$ were obtained in good yields.
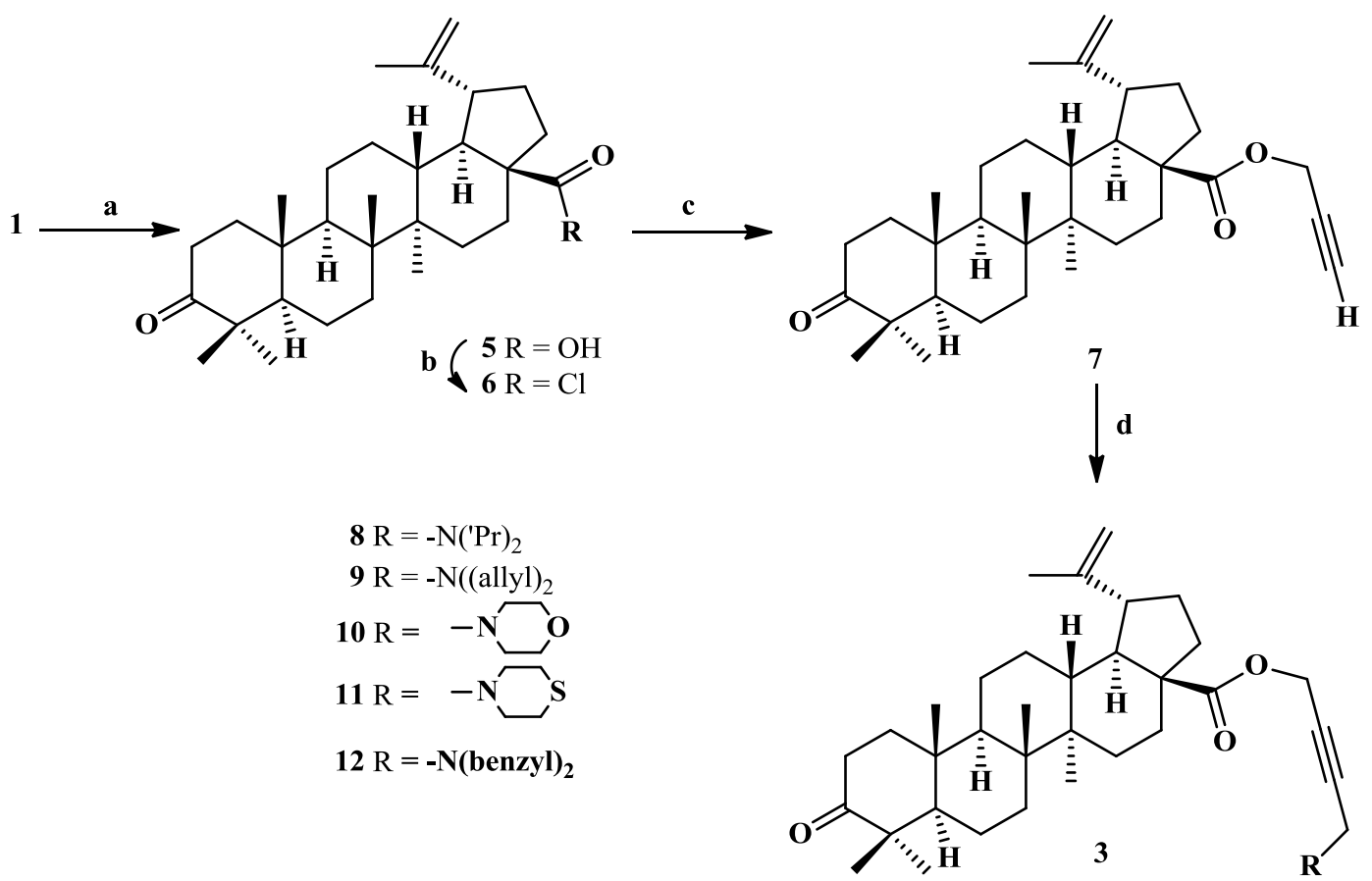

Scheme 1. Synthesis of Mannich bases 8-12: a) $\mathrm{CrO}_{3}, \mathrm{H}_{2} \mathrm{SO}_{4}$, acetone, $93 \%$ [ref. 15, 16]; b) (COCl) $)_{2}, \mathrm{DCM}, 25$ ${ }^{\circ} \mathrm{C}, 1 \mathrm{~h}$ (in-situ); c) propargylic alcohol, DCM, $25{ }^{\circ} \mathrm{C}, 5 \mathrm{~h}, 70 \%$; d) amine, formalin, CuI, DMSO, $40{ }^{\circ} \mathrm{C}, 72 \mathrm{~h}$, then dry $\mathrm{HCl}_{\mathrm{g}}$ in $\mathrm{Et}_{2} \mathrm{O}: \mathbf{8}(75 \%), 9(64 \%), \mathbf{1 0}(71 \%), \mathbf{1 1}(61 \%), \mathbf{1 2}(81 \%)$. 
To evaluate the influence of substitution at positions C-3 vs position C-28, several analogues derived from betulonate were prepared. To access these compounds, $\mathbf{1}$ was oxidized to betulonic acid $\mathbf{5}$ (Scheme 2) that was transformed into its methyl ester $\mathbf{1 3}^{20}$. Reaction of 13 with ethynylmagnesium bromide gave alkynol $\mathbf{1 4}^{14}$. From the $\mathrm{CuI}$ catalyzed Mannich reaction ${ }^{17,21}$ of $\mathbf{1 4}$ product $\mathbf{1 5}$ was obtained. From an acetylation of $\mathbf{1}$ the mono-acetate $\mathbf{1 6}$ was obtained, whose Swern oxidation furnished compound $\mathbf{1 7}^{22}$. This compound was transformed into the alkynol $\mathbf{1 8}$ as described above, and subjected to a Mannich reaction with diisopropylamine, formalin and $\mathrm{CuI}$ to yield $\mathbf{1 9}$ in $74 \%$ yield.

For comparison, similar compounds were prepared from glycyrrhetinic acid 4 (Scheme 3).
Thus 4 was converted into the methylester $\mathbf{2 0}^{23}$ and benzylester $21^{24}$. Oxidation of these esters gave the ketones $\mathbf{2 2}^{25}$ and $\mathbf{2 3}^{25}$. The reaction of $\mathbf{2 2}$ or $\mathbf{2 3}$ with ethynylmagnesium bromide furnished the alkynols $\mathbf{2 4}^{26-33}$ and 25, respectively. From the reaction of $\mathbf{2 4}$ or 25 with diallylamine or diisopropylamine and formalin in the presence of $\mathrm{CuI}$ the Mannich bases 26-28 were obtained in fair to good yields.

To explore the cytotoxic activity of the compounds, photometric sulforhodamin B assays $(\mathrm{SRB})^{34}$ were performed, and the $\mathrm{EC}_{50}$ values were determined. The results from these assays employing nine different human cancer cell lines and nonmalignant mouse fibroblasts are compiled in Table 1.

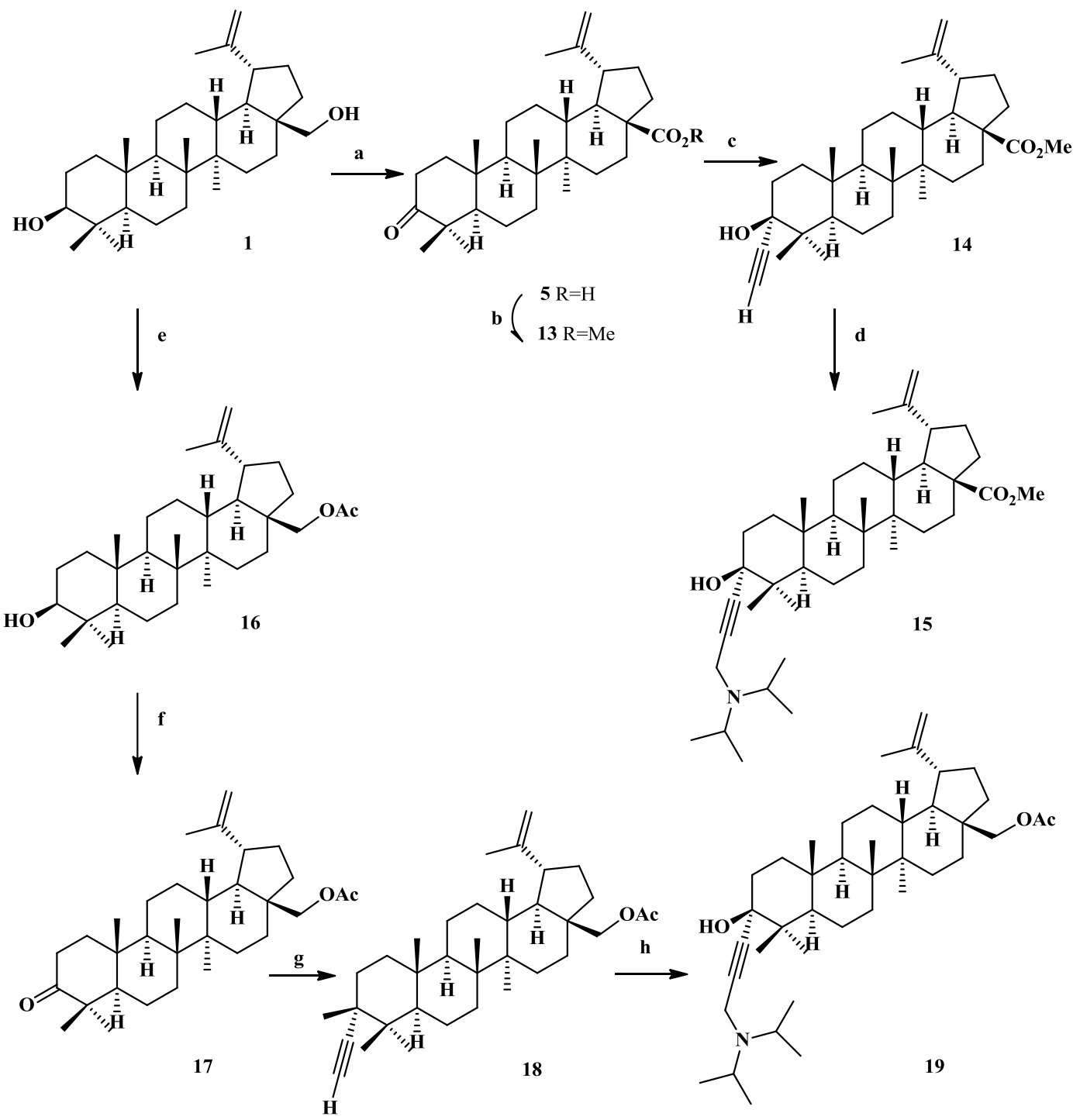

Scheme 2. Synthesis of Mannich bases 15 and 19: a) $\mathrm{CrO}_{3}, \mathrm{H}_{2} \mathrm{SO}_{4}$, acetone, $93 \%$ [ref. 15, 16], b) $(\mathrm{COCl})_{2}, 25$ ${ }^{\circ} \mathrm{C}, 1 \mathrm{~h}$, in-situ, then $\mathrm{MeOH}, 25^{\circ} \mathrm{C}, 4 \mathrm{~h}, 86 \%$; c) ethynylmagnesium bromide, THF, $25{ }^{\circ} \mathrm{C}, 72 \mathrm{~h}, 68 \%$; d) ('Prop) ${ }_{2} \mathrm{NH}$, formalin, CuI, DMSO, $40{ }^{\circ} \mathrm{C}, 72 \mathrm{~h}, 48 \%$; e) $\mathrm{Ac}_{2} \mathrm{O}, \mathrm{NEt}_{3}, \mathrm{DMAP} \mathrm{DCM}, 25^{\circ} \mathrm{C}, 6 \mathrm{~h}, 73 \%$; f) $(\mathrm{COCl})_{2}, \mathrm{DMSO}, \mathrm{NEt}_{3}, 81 \%$; g) ethynylmagnesium bromide, THF, $25{ }^{\circ} \mathrm{C}, 72 \mathrm{~h}, 75 \%$; h)

('Prop) ${ }_{2} \mathrm{NH}$, formalin, CuI, DMSO, $40{ }^{\circ} \mathrm{C}, 72 \mathrm{~h}, 74 \%$. 
As compared to parent compound betulinic acid $\mathbf{2}$, a significantly reduced cytotoxicity was observed for the ester $\mathbf{7}$ as well as for the Mannich bases derived from this ester (8-10). Also, alkynol 14 showed a slightly diminished cytotoxicity while the Mannich base $\mathbf{1 5}$ was shown to be approximately twice as cytotoxic as its parent compound 14. A reduced cytotoxicity was also observed for alkynol 18 and its Mannich base 19. For glycyrrhetinic acid derivatives, the alkynols $\mathbf{2 4}$ and $\mathbf{2 5}$ gave $\mathrm{EC}_{50}$ values in the same range as betulinic acid but they showed a significantly improved solubility in aqueous media. The Mannich bases 26-28, however, exhibited $\mathrm{EC}_{50}$ values approximately half of standard betulinic acid. For the most active compound of this series, glycyrrhetinic acid-derived compound $\mathbf{2 7}$ some extra assays were performed to gain a deeper insight into its mode of action. Thus, the death of A549 lung epithelial carcinoma cells was investigated by fluorescence microscopy ${ }^{35-38}$ using an AO/PI (acridine orange/propidium iodide) assay.

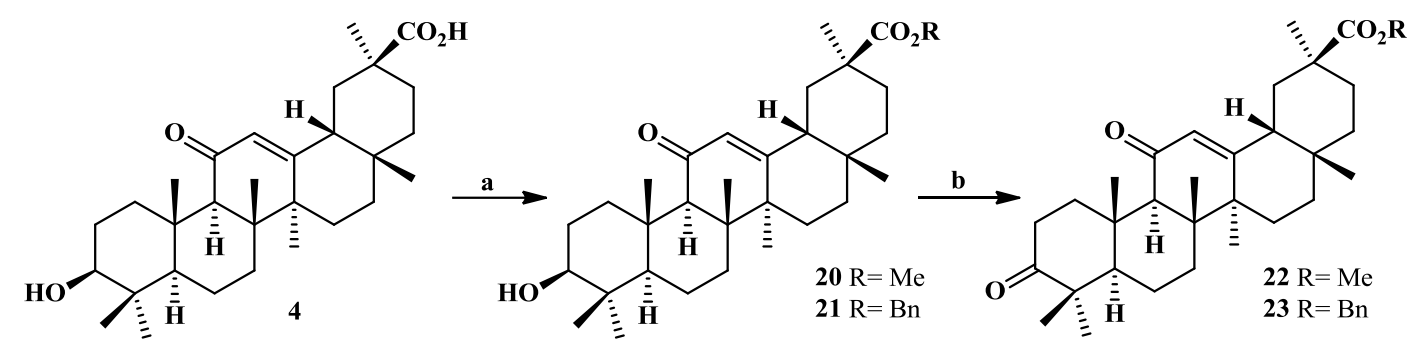

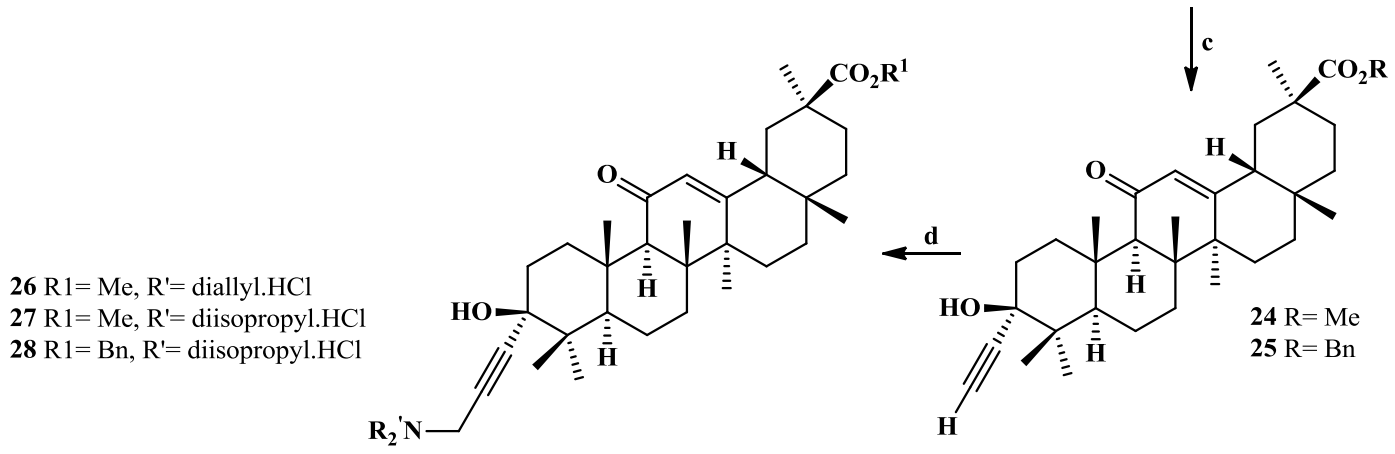

Scheme 3. Synthesis of Mannich bases 26-28: a) $\mathrm{K}_{2} \mathrm{CO}_{3}$, DMF, $25^{\circ} \mathrm{C}, 12 \mathrm{~h}, 97 \%$ (with MeI for 20), 75\% (with $\mathrm{BnBr}$ for 21); b) $(\mathrm{COCl})_{2}, \mathrm{DMSO}, \mathrm{NEt}_{3}, 87 \%$ (for 22), $\mathrm{CrO}_{3}, \mathrm{H}_{2} \mathrm{SO}_{4}$, acetone $87 \%$ (for 23); c) ethynylmagnesium bromide, THF, $25{ }^{\circ} \mathrm{C}, 72 \mathrm{~h}, 86 \%$ (for 24), 75\% (for 25); d) amine, CuI, DMSO, $40{ }^{\circ} \mathrm{C}, 72 \mathrm{~h}$, then dry $\mathrm{HCl}_{\mathrm{g}}$ in $\mathrm{Et}_{2} \mathrm{O}: 81 \%$ (with diallylamine for 26), 55\% (with diisopropylamine for 27 ), $63 \%$

(with diisopropylamine for $\mathbf{2 8}$ ).

The cells were treated for $48 \mathrm{~h}$ with betulinic acid 2 or compound 27. The presence of green fluorescent cells in this assay is a hallmark of apoptosis while the presence of orange colored cells indicates that they have died by a process of (secondary) necrosis. Hence, the results from this assay indicate that these cells had died from a controlled cell death: In addition, a DNA laddering experiment was performed. DNA laddering is a feature that is observed when DNA fragments resulting from an apoptotic DNA fragmentation by caspase-activated DNAse are detected after separation by gel electrophoresis. In this process DNA is cleaved by caspase-activated DNAse at internucleosomal linker regions, and DNA fragments of multiples of 180-185 base-pairs are formed. The experiments showed the formation of characteristic "DNA ladders" that are also a hallmark for apoptotic cell death.

Table 1: Cytotoxicity $\left(\mathrm{EC}_{50}\right.$ in $\left.\mu \mathrm{M}\right)$ of selected compounds measured in SRB-assays with different human cancer cell lines 518A2 (melanoma), 8505C (thyroid carcinoma), A253 (submandibular carcinoma), A549 (lung epithelial carcinoma), A2780 (ovarian carcinoma), DLD-1 (colon adenocarcinoma), LIPO (lipocarcinoma), MCF-7 (breast adenocarcinoma), SW1736 (thyroid carcinoma) and non-malignant mouse fibroblasts (NiH 3T3) in comparison with betulinic acid 2 . The $\mathrm{EC}_{50}$ values represent mean values obtained from three independent measurements each performed in triplicate (cut-off $30 \mu \mathrm{M}$ ). The values were obtained from the SRB assays after $96 \mathrm{~h}$ treatment of the cells with all the compounds. The values were averaged from at least 5 independent experiments and calculated applying the two-parametric Hill slope equation. 


\begin{tabular}{|c|c|c|c|c|c|c|c|c|c|c|}
\hline & 518A2 & $8505 \mathrm{C}$ & A253 & A549 & A2780 & DLD-1 & LIPO & MCF-7 & SW1736 & NIH 3T3 \\
\hline \multirow[t]{2}{*}{2} & 11.9 & 6.7 & 11.1 & 14.8 & 11.0 & 17.5 & 9.7 & 14.9 & 11.6 & 10.0 \\
\hline & \pm 0.7 & \pm 0.1 & \pm 0.3 & \pm 0.4 & \pm 0.4 & \pm 0.6 & \pm 0.2 & \pm 0.9 & \pm 0.7 & \pm 0.6 \\
\hline \multirow[t]{2}{*}{7} & 19.3 & 19.4 & 22.3 & 28.1 & 10.2 & 22.3 & $>30$ & 14.9 & 11.6 & 10.0 \\
\hline & \pm 1.0 & \pm 1.1 & \pm 0.9 & \pm 1.2 & \pm 1.2 & \pm 1.7 & & \pm 1.7 & \pm 1.3 & \pm 1.5 \\
\hline \multirow[t]{2}{*}{8} & 22.1 & 15.9 & 14.7 & $>30$ & 13.1 & 27.9 & $>30$ & 23.3 & 28.1 & 22.2 \\
\hline & \pm 1.6 & \pm 2.0 & \pm 1.1 & & \pm 0.7 & \pm 2.3 & & \pm 0.9 & \pm 1.9 & \pm 1.8 \\
\hline 9 & $>30$ & $>30$ & $>30$ & $>30$ & $>30$ & $>30$ & $>30$ & $>30$ & $>30$ & $>30$ \\
\hline \multirow[t]{2}{*}{10} & 17.1 & 14.3 & 13.9 & $>30$ & 13.0 & 19.0 & $>30$ & 13.3 & 22.8 & 19.1 \\
\hline & \pm 1.1 & \pm 1.0 & \pm 2.1 & & \pm 0.9 & \pm 1.3 & & \pm 1.7 & \pm 1.8 & \pm 2.3 \\
\hline \multirow[t]{2}{*}{14} & 20.0 & 21.9 & 13.0 & 21.8 & 26.8 & 16.1 & 22.8 & 27.8 & 20.8 & 9.4 \\
\hline & \pm 1.8 & \pm 2.3 & \pm 0.2 & \pm 0.9 & \pm 1.4 & \pm 2.5 & \pm 2.3 & \pm 2.8 & \pm 2.1 & \pm 0.8 \\
\hline \multirow[t]{2}{*}{15} & 5.7 & 5.6 & 5.6 & 7.2 & 7.4 & 7.3 & 8.5 & 5.1 & 6.3 & 3.7 \\
\hline & \pm 1.2 & \pm 0.8 & \pm 0.7 & \pm 0.6 & \pm 0.9 & \pm 0.4 & \pm 0.7 & \pm 1.0 & \pm 0.1 & \pm 0.5 \\
\hline \multirow[t]{2}{*}{16} & 20.9 & 21.0 & 19.1 & $>30$ & 16.6 & 21.2 & $>30$ & 19.5 & 23.9 & 27.6 \\
\hline & \pm 1.1 & \pm 2.1 & \pm 1.9 & & \pm 2.3 & \pm 1.7 & & \pm 2.0 & \pm 2.5 & \pm 2.1 \\
\hline \multirow[t]{2}{*}{17} & 10.8 & 11.6 & $>30$ & 13.7 & 5.9 & 7.3 & 9.7 & 6.8 & 18.8 & 10.1 \\
\hline & \pm 1.1 & \pm 0.9 & & \pm 0.5 & \pm 0.1 & \pm 0.5 & \pm 0.4 & \pm 0.3 & \pm 1.3 & \pm 0.9 \\
\hline \multirow[t]{2}{*}{18} & 24.0 & 15.2 & 17.1 & $>30$ & 11.4 & 21.3 & $>30$ & 22.4 & 22.3 & 27.1 \\
\hline & \pm 1.9 & \pm 0.9 & \pm 2.3 & & \pm 2.1 & \pm 2.3 & & \pm 2.5 & \pm 1.7 & \pm 2.2 \\
\hline \multirow[t]{2}{*}{19} & 17.6 & 13.4 & 11.3 & $>30$ & 10.2 & 15.2 & $>30$ & 15.1 & 18.3 & 18.9 \\
\hline & \pm 1.6 & \pm 0.7 & \pm 1.5 & & \pm 0.9 & \pm 1.5 & & \pm 1.8 & \pm 0.9 & \pm 2.1 \\
\hline \multirow[t]{2}{*}{24} & 23.0 & 12.7 & 13.3 & $>30$ & 6.1 & 16.2 & $>30$ & 12.4 & 17.3 & 20.9 \\
\hline & \pm 1.6 & \pm 0.8 & \pm 1.1 & & \pm 0.7 & \pm 1.3 & & \pm 1.3 & \pm 0.8 & \pm 1.9 \\
\hline \multirow[t]{2}{*}{25} & 7.8 & 9.9 & 10.4 & 15.4 & 14.3 & 10.1 & 8.3 & 10.3 & 10.4 & 10.9 \\
\hline & \pm 0.6 & \pm 0.5 & \pm 1.1 & \pm 1.9 & \pm 2.0 & \pm 1.5 & \pm 0.9 & \pm 1.6 & \pm 1.5 & \pm 1.1 \\
\hline \multirow[t]{2}{*}{26} & 7.5 & 8.4 & 8.8 & 11.4 & 6.8 & 8.6 & 7.3 & 9.1 & 9.9 & 5.8 \\
\hline & \pm 1.1 & \pm 1.3 & \pm 0.9 & \pm 1.6 & \pm 1.0 & \pm 0.8 & \pm 0.9 & \pm 0.7 & \pm 0.9 & \pm 1.2 \\
\hline \multirow[t]{2}{*}{27} & 5.0 & 3.4 & 3.3 & 7.1 & 7.1 & 6.3 & $>30$ & 4.9 & 7.1 & 3.5 \\
\hline & \pm 0.3 & \pm 0.7 & \pm 0.3 & \pm 0.4 & \pm 0.2 & \pm 0.1 & & \pm 0.8 & \pm 0.6 & \pm 0.7 \\
\hline \multirow[t]{2}{*}{28} & 6.5 & 4.1 & 5.1 & 8.6 & 8.9 & 6.2 & $>30$ & 5.9 & 8.1 & 5.3 \\
\hline & \pm 0.9 & \pm 0.6 & \pm 0.6 & \pm 0.5 & \pm 1.1 & \pm 0.7 & & \pm 0.5 & \pm 0.4 & \pm 0.9 \\
\hline
\end{tabular}

\section{Conclusion}

In summary, the formation of Mannich bases of betulinic or glycyrrhetinic acid derivatives improved their cytotoxicity. The highest cytotoxicity was determined for the 27, and $\mathrm{EC}_{50}$ values as low as 3.3 $\mu \mathrm{M}$ were determined for this compound employing the human submandibular carcinoma cell line A253. As proven by AO/PI staining as well as by DNAladdering experiments this compound acts by apoptosis.

\section{Acknowledgements}

We like to thank Dr. R. Kluge for the measurement of the ESI-MS spectra and Dr. D. Ströhl for the NMR spectra. The cell lines were kindly provided by Dr. Th. Müller (Dept. of Haematology/Oncology, Universität HalleWittenberg). Support by the "Gründerwerkstatt Biowissenschaften" is gratefully acknowledged. Ch. Otgonbayar wants to express his thanks to the German Academic Exchange Service (DAAD, Bonn) for a personal grant.

\section{Experimental Section}

Melting points are uncorrected (Leica hot stage microscope), NMR spectra were recorded using the Varian spectrometers Gemini 2000 or Unity $500(\delta$ given in ppm, $J$ in $\mathrm{Hz}$, internal $\mathrm{Me}_{4} \mathrm{Si}$; typical experiments: H-H-COSY, HMBC, HMQC, NOESY, DQF-COSY), ESI-MS spectra were taken on a Finnigan MAT LCQ 700 (electrospray, voltage $4.1 \mathrm{kV}$, sheath gas nitrogen) instrument. The optical rotation was measured on a Perkin-Elmer polarimeter at $20^{\circ} \mathrm{C}$; TLC was performed on silica gel (Merck 5554), column chromatography on silica gel (Merck KG60); elemental analyses were performed on a Vario EL (C-HNS). The solvents were dried according to usual procedures. The purity of the compounds were determined by HPLC and found to be $>98 \%$. Glycyrrhetinic acid was obtained from Orgentis Chemicals $\mathrm{GmbH}$ (Gatersleben) in bulk quantities.

The cultures of the cell were maintained as monolayer in RPMI 1640 (PAA Laboratories, Pasching, Germany) supplemented with $10 \%$ heat inactivated fetal bovine serum (Sigma AG, 
Germany) and penicillin/streptomycin (PAA Laboratories) at $37{ }^{\circ} \mathrm{C}$ in a humidified atmosphere of $5 \% \quad \mathrm{CO}_{2} / \quad 95 \%$ air. The cytotoxicity of the compounds was evaluated using the sulforhodamine-B (SRB) (Sigma Aldrich) ${ }^{34}$ microculture colorimetric assay as previously reported ${ }^{35-}$ 38. $E_{50}$ values were calculated from semi logarithmic dose response curves by non-linear regression applying a two parametrical Hill-slope equation. Values are given with a confidence interval $\mathrm{CI}=95 \%$.

The Acridine orange/ propidium iodide dye exclusion assay was performed as previously reported $^{35-38}$ employing approx. 500,000 cells (A2780). Visual analysis was performed under a fluorescence microscope. While green fluorescence showed apoptosis, a deep red colored nucleus indicated necrotic cells. The DNA laddering assay has been performed as previously described ${ }^{35-38}$.

\section{Extraction of betulin 1 and lupeol 3}

Dry birch bark (Betula platyphylla, $90 \mathrm{~g}$ ) was finely crushed and extracted with hexanes or diethylether for $15 \mathrm{~h}$ in a Soxhlet apparatus. The solvent was removed, and impure material was obtained $(9.45 \mathrm{~g}(10.5 \%)$ from the hexane extraction and $24.5 \mathrm{~g}(27.2 \%)$ from the diethylether extraction). By chromatographic work up (silica gel, toluene/ethyl acetate/heptane/formic acid, $80 / 20 / 10 / 3)$ from the hexane extract betulin $(4.4 \mathrm{~g}$, $47 \%)$ and lupeol (4.06 g, 43\%) were obtained, while the diethylether extract consisted of lupeol $(1.2 \mathrm{~g}$, $5 \%)$ and betulin $(19.8 \mathrm{~g}, 81 \%)$.

\section{Betulonic acid 5}

Betulonic acid was prepared from betulin 1 (18 $\mathrm{g}, 0.04 \mathrm{~mol})$ by Jones oxidation as previously described $^{15} ; 5$ (17.02 g, 93\%) was obtained as a colorless solid; $\mathrm{mp} 243-246{ }^{\circ} \mathrm{C}$ (lit.: $245-247^{15}$ ); $[\alpha]_{\mathrm{D}}=36.7^{\circ}\left(c=6.20, \mathrm{CHCl}_{3}\right)$ (lit.: $45^{\circ}(c=5.1$, $\left.\mathrm{CHCl}_{3}\right)^{15} ; \mathrm{R}_{F}=0.51$ ( $n$-hexane/ethyl acetate, 8/2).

Prop-2-yn-1-yl 3-oxolup-20(29)-en-28-oate 7

Oxalyl chloride $(1.4 \mathrm{~mL}, 16.3 \mathrm{mmol})$ was added to a solution of $5(3.7 \mathrm{~g}, 8.13 \mathrm{mmol})$ in anhydrous DCM $(10 \mathrm{~mL})$, and the mixture was stirred at $25{ }^{\circ} \mathrm{C}$ for 5 hours. The volatiles were evaporated under reduced pressure, the residue (consisting of acid chloride 6) was dissolved in anhydrous DCM $(10 \mathrm{~mL})$, evaporated (dissolving in DCM and evaporation was repeated $4 \mathrm{x}$ ) and finally dissolved in anhydrous THF (20 mL). Prop-2-yn-1-ol (1 mL, $18 \mathrm{mmol}$ ) was added, and stirring at $25{ }^{\circ} \mathrm{C}$ was continued for another 3 hours. The solvent was removed, and the residue was subjected to column chromatography $\left(\mathrm{SiO}_{2}\right.$, hexanes/ ethyl acetate, $\left.8 / 2\right)$ to yield $7^{18,19}(2.8 \mathrm{~g}, 70 \%)$ as a colorless solid; mp $162-165{ }^{\circ} \mathrm{C} ;[\alpha]_{\mathrm{D}}=29.5^{\circ}\left(c=5.3, \mathrm{CHCl}_{3}\right) ; \mathrm{R}_{F}=0.64$ (n-hexane/ethyl aceate, $8 / 2) ;{ }^{1} \mathrm{H}$ NMR $(500 \mathrm{MHz}$, $\left.\mathrm{CDCl}_{3}\right): \delta=4.77(d, 1 \mathrm{H}, J=2.1 \mathrm{~Hz}, \mathrm{H}-29 \mathrm{a}), 4.74$ $(d d, 1 \mathrm{H}, J=2.4,15.5 \mathrm{~Hz}, \mathrm{H}-31 \mathrm{a}), 4.66(d d, 1 \mathrm{H}$,
$J=2.5,15.5 \mathrm{~Hz}, \mathrm{H}-31 \mathrm{~b}), 4.64(d d, 1 \mathrm{H}, J=1.4,2.1$ $\mathrm{Hz}, \mathrm{H}-29 \mathrm{~b}), 3.05$ (ddd, $1 \mathrm{H}, J=4.8,10.9,10.9 \mathrm{~Hz}$, $\mathrm{H}-19), 2.51$ ( $d d d, 1 \mathrm{H}, J=7.6,9.8,15.7, \mathrm{H}-2 \mathrm{a}), 2.46$ $(t, 1 \mathrm{H}, J=2.4 \mathrm{~Hz}, \mathrm{H}-33), 2.42$ (ddd, $1 \mathrm{H}, J=4.4,7.6$, $15.6 \mathrm{~Hz}, \mathrm{H}-2 \mathrm{~b}), 2.33-2.29$ ( $m, 1 \mathrm{H}, \mathrm{H}-16), 2.28-2.24$ $(m, 1 \mathrm{H}, \mathrm{H}-13), 1.99-1.89$ (m, 3H, H-1a $+\mathrm{H}-21 \mathrm{a}+$ $\mathrm{H}-22 \mathrm{a}), 1.78-1.72(m, 1 \mathrm{H}, \mathrm{H}-12), 1.71(s, 3 \mathrm{H}, \mathrm{H}-30)$, $1.65(t, 1 \mathrm{H}, J=11.4 \mathrm{~Hz}, \mathrm{H}-18), 1.52-1.26(m, 13 \mathrm{H}$, $\mathrm{H}-9+\mathrm{H}-21 \mathrm{~b}+\mathrm{H}-16+\mathrm{H}-1 \mathrm{~b}+\mathrm{H}-7+\mathrm{H}-6+\mathrm{H}-5+$ $\mathrm{H}-22 \mathrm{~b}+\mathrm{H}-15 \mathrm{a}+\mathrm{H}-11), 1.24-1.20$ ( $m, 1 \mathrm{H}, \mathrm{H}-15 \mathrm{~b})$, 1.09 ( $s, 3 \mathrm{H}, \mathrm{H}-24), 1.08-1.05$ ( $m, 1 \mathrm{H}, \mathrm{H}-12 \mathrm{~b}), 1.04$ $(s, 3 \mathrm{H}, \mathrm{H}-27), 1.01(s, 3 \mathrm{H}, \mathrm{H}-26), 0.99(s, 3 \mathrm{H}, \mathrm{H}-25)$, 0.95 (s, 3H, H-23) ppm; ${ }^{13} \mathrm{C} \mathrm{NMR}(500 \mathrm{MHz}$, $\left.\mathrm{CDCl}_{3}\right): \delta=218.0(\mathrm{C}-3), 175.1$ (C-28), 150.3 (C-20), 109.7 (C-29), $78.1 \quad(\mathrm{C}-32, C \equiv \mathrm{CH}), 74.3$ (C-33, $\mathrm{C} \equiv C \mathrm{H}), 56.5$ (C-17), 55.0 (C-5), 51.3 (C-31), 49.9 (C-18), 49.4 (C-9), 47.3 (C-4), 46.8 (C-19), 42.5 (C-8), 40.7 (C-14), 39.6 (C-1), 38.3 (C-13), 36.9 (C-2), 36.7 (C-10), 34.1 (C-22), 33.6 (C-7), 31.8 (C-16), 30.5 (C-21), 29.6 (C-15), 26.6 (C-23), 25.5 (C-12), 21.4 (C-11), 21.0 (C-30), 19.6 (C-6), 19.4 (C-24), 15.9 (C-26), 15.8 (C-25), 14.6 (C-27) ppm; MS (ESI, MeOH): $m / z=493.3\left(53 \%,[\mathrm{M}+\mathrm{H}]^{+}\right)$, $547.0\left(100 \%,[\mathrm{M}+\mathrm{Na}+\mathrm{MeOH}]^{+}\right), 1007.2(66 \%$, $\left.[2 \mathrm{M}+\mathrm{Na}]^{+}\right)$; analysis calculated for $\mathrm{C}_{33} \mathrm{H}_{48} \mathrm{O}_{3}$ (492.73) C 80.44, H 9.82; found: C 80.31, H 10.02.

\section{3-Diisopropylaminoprop-2-yn-1-yl 3-oxolup- 20(29)-en-28-oate hydrochloride 8}

Compound 7 (200 mg, $0.41 \mathrm{mmol})$ was dissolved in THF $(10 \mathrm{~mL})$ and allowed to react at 40 ${ }^{\circ} \mathrm{C}$ with diisopropylamine (126 $\left.\mu \mathrm{l}, 0.9 \mathrm{mmol}\right)$, formalin $(37 \%, 0.16 \mathrm{~mL}, 2 \mathrm{mmol})$ and $\mathrm{CuI}(20 \mathrm{mg}$, $0.1 \mathrm{mmol}$ ) for 72 hours. The volatiles were removed under reduced pressure, the residue was dissolved in DCM $(50 \mathrm{~mL})$, washed with ammonium hydroxide (aq., $0.05 \mathrm{M}$ ) and water, dried $\left(\mathrm{Na}_{2} \mathrm{SO}_{4}\right)$, the solvent was evaporated, and the residue subjected to column chromatography $\left(\mathrm{SiO}_{2}\right.$, hexanes/ethyl acetate, $8 / 2$; methanol). Fractions containing the product were collected, the solvent was evaporated, the residue was dissolved in diethyl ether, and dry $\mathrm{HCl}$ gas was passed through for several minutes. After standing at $4{ }^{\circ} \mathrm{C}$ for 12 hours, the precipitate was filtered off, washed with water and diethyl ether to afford 8 (198 $\mathrm{mg}, 75 \%)$ as a colorless solid; $\mathrm{mp} 171-173{ }^{\circ} \mathrm{C}$; $[\alpha]_{\mathrm{D}}=46.2^{\circ}(c=5.6, \mathrm{MeOH}) ;{ }^{1} \mathrm{H} \mathrm{NMR}(400 \mathrm{MHz}$, $\left.\mathrm{CDCl}_{3}\right): \delta=4.66(d, 1 \mathrm{H}, J=2.1 \mathrm{~Hz}, \mathrm{H}-29 \mathrm{a}), 4.63-$ $4.60(m, 2 \mathrm{H}, \mathrm{H}-31), 4.54(d d, 1 \mathrm{H}, J=1.4,2.2 \mathrm{~Hz}$, $\mathrm{H}-29 \mathrm{~b}), 3.45-3.40$ ( $m, 2 \mathrm{H}, \mathrm{H}-34), 3.16$ (ddd, 2H, $J=6.5,6.5,13.1 \mathrm{~Hz}, \mathrm{H}-35), 2.98-2.88(m, 1 \mathrm{H}, \mathrm{H}-$ 19), 2.42 ( $d d d, 1 \mathrm{H}, J=7.5,9.8,15.8 \mathrm{~Hz}, \mathrm{H}-2 \mathrm{a}), 2.32$ (ddd, $1 \mathrm{H}, J=4.4,7.5,15.6 \mathrm{~Hz}, \mathrm{H}-2 \mathrm{~b}), 2.23-2.13$ ( $m$, $2 \mathrm{H}, \mathrm{H}-13+\mathrm{H}-21 \mathrm{a}), 1.89-1.77(m, 3 \mathrm{H}, \mathrm{H}-22 \mathrm{a}+\mathrm{H}-16$ $+\mathrm{H}-1 \mathrm{a}), 1.69-1.62(m, 1 \mathrm{H}, \mathrm{H}-12), 1.62(s, 3 \mathrm{H}$, $\mathrm{H}-30), 1.54(t, 1 \mathrm{H}, J=11.4 \mathrm{~Hz}, \mathrm{H}-18), 1.44-1.08$ $(m, 16 \mathrm{H}, \mathrm{H}-9+\mathrm{H}-21 \mathrm{~b}+\mathrm{H}-16+\mathrm{H}-1 \mathrm{~b}+\mathrm{H}-22 \mathrm{~b}+\mathrm{H}-$ $7+\mathrm{H}-6+\mathrm{H}-5+\mathrm{H}-15+\mathrm{H}-11), 1.06(s, 6 \mathrm{H}, \mathrm{H}-36)$, $1.04(s, 6 \mathrm{H}, \mathrm{H}-36), 1.00$ ( $s, 3 \mathrm{H}, \mathrm{H}-24), 0.95(s, 3 \mathrm{H}$, H-27), 0.94-0.93 ( $m, 1 \mathrm{H}, \mathrm{H}-12), 0.90$ ( $s, 3 \mathrm{H}, \mathrm{H}-26)$, $0.89(s, 3 \mathrm{H}, \mathrm{H}-25), 0.85(s, 3 \mathrm{H}, \mathrm{H}-23) \mathrm{ppm} ;{ }^{13} \mathrm{C}$ 
NMR (125 MHz, $\left.\mathrm{CDCl}_{3}\right): \delta=218.0(\mathrm{C}-3), 175.3$ (C-28), 150.4 (C-20), 109.7 (C-29), 85.4 (C-32, $C \equiv \mathrm{C}), 77.9$ (C-33, $\mathrm{C} \equiv C), 56.6(\mathrm{C}-17), 55.0(\mathrm{C}-5)$, 52.0 (C-31), 49.9 (C-9), 49.4 (C-18), 48.8 (C-35), 47.3 (C-4), 46.9 (C-19), 42.4 (C-8), 40.6 (C-14), 39.6 (C-1), $38.3 \quad$ (C-13), 36.9 (C-10), 36.8 (C-22), 34.4 (C-34), 34.1 (C-2), 33.6 (C-7), 31.9 (C-21), 30.5 (C-16), 29.6 (C-15), 26.6 (C-23), 25.5 (C-12), 21.4 (C-11), 20.4 (C-36, 4 x $\left.\mathrm{CH}_{3}\right), 19.6$ (C-6), 19.3 (C-30), 15.9 (C-24 + C-26), 15.7 (C-25), 14.6 (C-27) ppm; MS (ESI, MeOH): $m / z=606.6(100 \%$, $\left.[\mathrm{M}+\mathrm{H}]^{+}\right)$; analysis calculated for $\mathrm{C}_{40} \mathrm{H}_{64} \mathrm{ClNO}_{3}$ (642.46): C 74.79, H 10.04, N 2.18; found: C 74.61, H 10.27, N 2.13.

\section{3-Diallylaminoprop-2-yn-1-yl 3-oxolup- 20(29)-en-28-oate hydrochloride 9}

Following the procedure for the synthesis of $\mathbf{8}$, from 7 (200 mg, $0.41 \mathrm{mmol})$, diallylamine $(110 \mu \mathrm{L}$, $0.9 \mathrm{mmol})$, formalin $(37 \%, 0.16 \mathrm{~mL}, 2 \mathrm{mmol})$ and CuI (20 mg, $0.1 \mathrm{mmol}) 9$ (194 mg (64\%) was obtained as a colorless solid; $\mathrm{mp} 195-198{ }^{\circ} \mathrm{C} ;[\alpha]_{\mathrm{D}}=$ $36.8^{\circ}(c=5.5, \mathrm{MeOH}) ;{ }^{1} \mathrm{H} \mathrm{NMR}(500 \mathrm{MHz}$, $\left.\mathrm{CD}_{3} \mathrm{OD}\right): \delta=6.08-5.96(m, 2 \mathrm{H}, \mathrm{H}-36), 5.75-5.64(m$, $4 \mathrm{H}, \mathrm{H}-37), 4.90-4.87$ ( $m, 2 \mathrm{H}, \mathrm{H}-31), 4.74(d, 1 \mathrm{H}$, $J=1.6 \mathrm{~Hz}, \mathrm{H}-29 \mathrm{a}), 4.64(d d, 1 \mathrm{H}, J=1.4,2.2 \mathrm{~Hz}$, $\mathrm{H}-29 \mathrm{~b}), 4.16-4.14$ ( $m, 2 \mathrm{H}, \mathrm{H}-34), 3.96-3.89$ ( $m, 4 \mathrm{H}$, $\mathrm{H}-35), 3.02$ (ddd, 1H, $J=4.9,10.9,10.9 \mathrm{~Hz}, \mathrm{H}-19)$, 2.35-2.25 (m, 2H, H-13 + H-21a), 1.94-1.82 (m, 3H, $\mathrm{H}-22 \mathrm{a}+\mathrm{H}-16+\mathrm{H}-1 \mathrm{a}), 1.80-1.75\left(m, 2 \mathrm{H}, \mathrm{CH}_{2}(12)\right)$, $1.72(s, 3 \mathrm{H}, \mathrm{H}-30), 1.59-1.06(m, 15 \mathrm{H}, \mathrm{H}-9+\mathrm{H}-18+$ $\mathrm{H}-21 \mathrm{~b}+\mathrm{H}-16+\mathrm{H}-1 \mathrm{~b}+\mathrm{H}-22 \mathrm{~b}+\mathrm{H}-7+\mathrm{H}-6+\mathrm{H}-5+$ $\mathrm{H}-15+\mathrm{H}-11), 1.05$ ( $s, 3 \mathrm{H}, \mathrm{H}-24), 1.03$ ( $s, 3 \mathrm{H}, \mathrm{H}-27)$, $1.01(s, 3 \mathrm{H}, \mathrm{H}-26), 0.98(s, 3 \mathrm{H}, \mathrm{H}-25), 0.93(s, 3 \mathrm{H}$, $\mathrm{H}-23) \mathrm{ppm} ;{ }^{13} \mathrm{C} \mathrm{NMR}\left(125 \mathrm{MHz}, \mathrm{CDCl}_{3}\right): \delta=218.0$ (C-3), 175.2 (C-28), 150.1 (C-20), 126.6 (C-37),

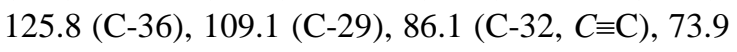
$(\mathrm{C}-33, \mathrm{C} \equiv C), 56.6(\mathrm{C}-17), 54.8(\mathrm{C}-35), 54.6(\mathrm{C}-5)$, 50.9 (C-31), 49.7 (C-18), 49.2 (C-9), 47.1 (C-19), 46.9 (C-4), 42.3 (C-8), 41.0 (C-34), 40.5 (C-14), 39.2 (C-1), 38.4 (C-13), 36.7 (C-10), 36.3 (C-22), 33.3 (C-7), 31.4 (C-21), 30.2 (C-16), 29.4 (C-15), 25.8 (C-23), 25.4 (C-12), 21.2 (C-11), 19.8 (C-30), 19.4 (C-6), 18.1 (C-24), 15.2 (C-26), 15.1 (C-25), 13.7 (C-27) ppm; MS (ESI, MeOH): $m / z=602.6$ $\left(100 \%, \quad[\mathrm{M}+\mathrm{H}]^{+}\right)$; analysis calculated for $\mathrm{C}_{40} \mathrm{H}_{60} \mathrm{ClNO}_{3}$ (638.36): C 75.26, H 9.47, N 2.19; found: C 75.13, H 9.65, N 2.11.

3-Morpholin-4-yl-prop-2-yn-1-yl 3-oxolup20(29)-en-28-oate hydrochloride 10

Following the procedure given for the synthesis of $\mathbf{8}$, from 7 ( $200 \mathrm{mg}, 0.41 \mathrm{mmol})$, morpholine (39 $\mathrm{mL}, 4.46 \mathrm{mmol})$, formalin $(37 \%, 0.16 \mathrm{~mL}, 2 \mathrm{mmol})$ and $\mathrm{CuI}(20 \mathrm{mg}, 0.1 \mathrm{mmol}) \mathbf{1 0}(183 \mathrm{mg}, 71 \%)$ was obtained as a colorless solid; $\mathrm{mp} 153-155^{\circ} \mathrm{C} ;[\alpha]_{\mathrm{D}}=$ $18.5^{\circ}(c=3.45, \mathrm{MeOH}) ;{ }^{1} \mathrm{H}$ NMR $(400 \mathrm{MHz}$, $\left.\mathrm{CD}_{3} \mathrm{OD}\right): \delta=4.75-4.72(m, 2 \mathrm{H}, \mathrm{H}-31), 4.62(d, 1 \mathrm{H}$, $J=1.4 \mathrm{~Hz}, \mathrm{H}-29 \mathrm{a}), 4.54-4.50$ ( $m, 1 \mathrm{H}, \mathrm{H}-29 \mathrm{~b}), 4.13-$ 4.09 ( $m, 2 \mathrm{H}, \mathrm{H}-34), 4.06-3.94$ ( $m, 2 \mathrm{H}, \mathrm{H}-36 \mathrm{a}), 3.75-$ $3.62(m, 2 \mathrm{H}, \mathrm{H}-36 \mathrm{~b}), 3.53-3.38(m, 2 \mathrm{H}, \mathrm{H}-35 \mathrm{a})$, 3.31-3.10 ( $m, 2 \mathrm{H}, \mathrm{H}-35 \mathrm{~b}), 2.89$ (ddd, $1 \mathrm{H}, J=4.8$,
10.8, $10.8 \mathrm{~Hz}, \mathrm{H}-19), 2.24-2.12$ ( $m, 2 \mathrm{H}, \mathrm{H}-13+$ $\mathrm{H}-21 \mathrm{a}), 1.85-1.71(m, 3 \mathrm{H}, \mathrm{H}-22 \mathrm{a}+\mathrm{H}-16+\mathrm{H}-1 \mathrm{a})$, 1.69-1.57 ( $m, 5 \mathrm{H}, \mathrm{H}-12+\mathrm{H}-30+\mathrm{H}-18), 1.50-0.98$ $(m, 14 \mathrm{H}, \mathrm{H}-9+\mathrm{H}-21 \mathrm{~b}+\mathrm{H}-16+\mathrm{H}-1 \mathrm{~b}+\mathrm{H}-22 \mathrm{~b}+$ $\mathrm{H}-7+\mathrm{H}-6+\mathrm{H}-5+\mathrm{H}-15+\mathrm{H}-11), 0.97(s, 3 \mathrm{H}, \mathrm{H}-$ 24), 0.96-0.94 ( $m, 1 \mathrm{H}, \mathrm{H}-12 \mathrm{~b}), 0.94(s, 3 \mathrm{H}, \mathrm{H}-27)$, $0.92(s, 3 \mathrm{H}, \mathrm{H}-26), 0.90$ ( $s, 3 \mathrm{H}, \mathrm{H}-25), 0.85$ ( $s, 3 \mathrm{H}$, $\mathrm{H}-23) \mathrm{ppm} ;{ }^{13} \mathrm{C} \mathrm{NMR}\left(125 \mathrm{MHz}, \mathrm{CDCl}_{3}\right): \delta=219.5$ (C-3), 175.2 (C-28), 150.1 (C-20), 109.1 (C-29), $85.9(\mathrm{C}-32, C \equiv \mathrm{C}), 73.5(\mathrm{C}-33, \mathrm{C} \equiv C), 63.6(\mathrm{C}-36)$, 56.6 (C-17), 54.6 (C-5), 51.1 (C-35), 50.8 (C-31), 49.7 (C-9), 49.1 (C-18), 47.0 (C-19), 45.6 (C-4), 42.2 (C-8), 40.5 (C-14), 40.4 (C-34), 39.1 (C-1), 38.4 (C-13), 36.6 (C-10), 36.2 (C-22), 33.3 (C-7), 31.4 (C-21), 30.1 (C-16), 29.4 (C-15), 25.7 (C-23), 25.4 (C-12), 21.2 (C-11), 19.9 (C-30), 19.3 (C-6), 18.0 (C-24), 15.2 (C-26), 15.0 (C-25), 13.6 (C-27) ppm; MS (ESI, MeOH): $\mathrm{m} / \mathrm{z}=592.6(100 \%$, $\left.[\mathrm{M}+\mathrm{H}]^{+}\right)$; analysis calculated for $\mathrm{C}_{38} \mathrm{H}_{58} \mathrm{ClNO}_{4}$ (628.32): C 72.64, H 9.30, N 2.23; found: C 72.49, H 9.41, N 2.18.

\section{3-Thiomorpholin-4-yl-prop-2-yn-1-yl 3-} oxolup-20(29)-en-28-oate hydrochloride 11

Following the procedure for the synthesis of $\mathbf{8}$, from 7 (200 mg, $0.41 \mathrm{mmol})$, thiomorpholine (116 $\mu \mathrm{L}, 1.15 \mathrm{mmol})$, formalin $(37 \%, 0.16 \mathrm{~mL}, 2 \mathrm{mmol})$ and $\mathrm{CuI}(20 \mathrm{mg}, 0.1 \mathrm{mmol}) \mathbf{1 1}(147 \mathrm{mg}, 61 \%)$ was obtained as a colorless solid; $m p 173-178{ }^{\circ} \mathrm{C} ;[\alpha]_{\mathrm{D}}=$ $31.2^{\circ}\left(c=3.1, \mathrm{CHCl}_{3}\right) ;{ }^{1} \mathrm{H} \mathrm{NMR}\left(400 \mathrm{MHz}, \mathrm{CDCl}_{3}\right)$ : $\delta=4.67(d, 1 \mathrm{H}, J=2.0 \mathrm{~Hz}, \mathrm{H}-29 \mathrm{a}), 4.64(d t, 2 \mathrm{H}$, $J=1.9,9.3 \mathrm{~Hz}, \mathrm{H}-31), 4.54(d d, 1 \mathrm{H}, J=1.4,2.1 \mathrm{~Hz}$, H-29b), 3.25 ( $t, 2 \mathrm{H}, J=1.9 \mathrm{~Hz}, \mathrm{H}-34), 2.98-2.90$ ( $m, 1 \mathrm{H}, \mathrm{H}-19), 2.76-2.61$ ( $m, 8 \mathrm{H}, \mathrm{H}-35+\mathrm{H}-36), 2.42$ $(d d d, 1 \mathrm{H}, J=7.5,9.8,15.8 \mathrm{~Hz}, \mathrm{H}-2 \mathrm{a}), 2.32(d d d, 1 \mathrm{H}$, $J=4.4,7.5,15.7 \mathrm{~Hz}, \mathrm{H}-2 \mathrm{~b}), 2.24-2.14$ ( $m, 2 \mathrm{H}, \mathrm{H}-13$ $+\mathrm{H}-21 \mathrm{a}), 1.89-1.76(m, 3 \mathrm{H}, \mathrm{H}-22 \mathrm{a}+\mathrm{H}-16+\mathrm{H}-1 \mathrm{a})$, 1.69-1.63 ( $m, 1 \mathrm{H}, \mathrm{H}-12), 1.62(s, 3 \mathrm{H}, \mathrm{H}-30), 1.55$ $(t, 1 \mathrm{H}, J=11.4 \mathrm{~Hz}, \mathrm{H}-18), 1.45-1.01(m, 14 \mathrm{H}, \mathrm{H}-9+$ $\mathrm{H}-21 \mathrm{~b}+\mathrm{H}-16+\mathrm{H}-1 \mathrm{~b}+\mathrm{H}-22 \mathrm{~b}+\mathrm{H}-7+\mathrm{H}-6+\mathrm{H}-5+$ $\mathrm{H}-15+\mathrm{H}-11), 1.00$ ( $s, 3 \mathrm{H}, \mathrm{H}-24), 0.99-0.96(m, 1 \mathrm{H}$, $\mathrm{H}-12 \mathrm{~b}), 0.95$ ( $s, 3 \mathrm{H}, \mathrm{H}-27), 0.91(s, 3 \mathrm{H}, \mathrm{H}-26), 0.90$ $(s, 3 \mathrm{H}, \mathrm{H}-25), 0.85(s, 3 \mathrm{H}, \mathrm{H}-23) \mathrm{ppm} ;{ }^{13} \mathrm{C} \mathrm{NMR}$ $\left(125 \mathrm{MHz}, \mathrm{CDCl}_{3}\right): \delta=218.0(\mathrm{C}-3), 175.2(\mathrm{C}-28)$, 150.4 (C-20), 109.7 (C-29), 87.1 (C-32, $C \equiv \mathrm{C}), 79.4$ $(\mathrm{C}-33, \mathrm{C} \equiv C), 56.6(\mathrm{C}-17), 55.0(\mathrm{C}-5), 53.8(\mathrm{C}-35)$, 51.7 (C-31), 49.9 (C-9), 49.4 (C-18), 48.4 (C-34), 47.3 (C-4), 46.9 (C-19), 42.5 (C-8), 40.7 (C-14), 39.6 (C-1), 38.3 (C-13), 36.9 (C-10), 36.8 (C-22), 34.1 (C-2), 33.6 (C-7), 31.9 (C-21), 30.5 (C-16), 29.6 (C-15), 28.0 (C-36), 26.6 (C-23), 25.5 (C-12), 21.4 (C-11), 19.6 (C-6), 19.3 (C-30), 15.9 (C-24 + $\left.\mathrm{C} 26,2 \times \mathrm{CH}_{3}\right), 15.8(\mathrm{C}-25), 14.6(\mathrm{C}-27) \mathrm{ppm} ; \mathrm{MS}$ (ESI, $\mathrm{MeOH}): m / z=608.5\left(100 \%,[\mathrm{M}+\mathrm{H}]^{+}\right)$; analysis calculated for $\mathrm{C}_{38} \mathrm{H}_{58} \mathrm{ClNO}_{3} \mathrm{~S}$ (644.39): C 70.83, H 9.07, N 2.17, S 4.98; found: C 70.68, H 9.24, N 2.03, S 4.77 . 
[3-(Dibenzylamino)-prop-2-yn-1-yl] 3oxolup-20(29)-en-28-oate hydrochloride 12

Following the procedure for the synthesis of $\mathbf{8}$, from 7 (200 mg, $0.41 \mathrm{mmol})$, dibenzylamine $(20 \mu \mathrm{L}$, $4.46 \mathrm{mmol})$, formalin $(37 \%, 0.16 \mathrm{~mL}, 2 \mathrm{mmol})$ and $\mathrm{CuI}$ (20 mg, $0.1 \mathrm{mmol}) \mathbf{1 2}(245 \mathrm{mg}, 81 \%)$ was obtained as a colorless solid; $\mathrm{mp} 187-192{ }^{\circ} \mathrm{C} ;[\alpha]_{\mathrm{D}}=$ $18.6^{\circ}(c=3.6, \mathrm{MeOH}) ;{ }^{1} \mathrm{H}$ NMR $(400 \mathrm{MHz}$, $\left.\mathrm{CD}_{3} \mathrm{OD}\right): \delta=7.62-7.47(m, 10 \mathrm{H}, 10 \times \mathrm{CH}(\mathrm{Ph})), 4.97$ $(d, 1 \mathrm{H}, J=15.9 \mathrm{~Hz}, \mathrm{H}-31 \mathrm{a}), 4.90(d, 1 \mathrm{H}, J=16.0$ $\mathrm{Hz}, \mathrm{H}-31 \mathrm{~b}), 4.69(d, 1 \mathrm{H}, J=0.9 \mathrm{~Hz}, \mathrm{H}-29 \mathrm{a})$, 4.62-4.58 ( $m, 1 \mathrm{H}, \mathrm{H}-29 \mathrm{~b}), 4.55-4.46(m, 4 \mathrm{H}, \mathrm{H}-35)$, 3.88-3.84 ( $m, 2 \mathrm{H}, \mathrm{H}-34), 3.03$ (ddd, $1 \mathrm{H}, J=4.8$, $10.8,10.8 \mathrm{~Hz}, \mathrm{H}-19), 2.34-2.23$ ( $m, 2 \mathrm{H}, \mathrm{H}-13+$ $\mathrm{H}-21 \mathrm{a}), 1.99-1.89$ ( $m, 3 \mathrm{H}, \mathrm{H}-22 \mathrm{a}+\mathrm{H}-16+\mathrm{H}-1 \mathrm{a})$, 1.76-1.78 $(m, 5 \mathrm{H}, \mathrm{H}-12+\mathrm{H}-30+\mathrm{H}-18), 1.60-1.05$ $(m, 14 \mathrm{H}, \mathrm{H}-9+\mathrm{H}-21 \mathrm{~b}+\mathrm{H}-16+\mathrm{H}-1 \mathrm{~b}+\mathrm{H}-22 \mathrm{~b}+$ $\mathrm{H}-7+\mathrm{H}-6+\mathrm{H}-5+\mathrm{H}-15+\mathrm{H}-11), 1.04(s, 3 \mathrm{H}, \mathrm{H}-$ 24), 1.02 ( $s, 3 \mathrm{H}, \mathrm{H}-27), 1.01-0.99$ ( $m, 1 \mathrm{H}, \mathrm{H}-12 \mathrm{~b})$, $0.99(s, 3 \mathrm{H}, \mathrm{H}-26), 0.88(s, 3 \mathrm{H}, \mathrm{H}-25), 0.85(s, 3 \mathrm{H}$, $\mathrm{H}-23) \mathrm{ppm} ;{ }^{13} \mathrm{C}$ NMR $\left(125 \mathrm{MHz}, \mathrm{CD}_{3} \mathrm{OD}\right)$ : $\delta=219.5$ (C-3), 175.3 (C-28), 150.1 (C-20), 131.0 (Ph, 4 x CH), $130.2(\mathrm{Ph}, 2 \times \mathrm{CH}), 129.2(\mathrm{Ph}, 4 \times \mathrm{CH})$, $128.8\left(\mathrm{Ph}, 2 \times C_{\text {quart. }}\right), 109.1$ (C-29), 87.2 (C-32, $C \equiv \mathrm{C}), 73.5(\mathrm{C}-33, \mathrm{C} \equiv C), 56.9(\mathrm{C}-35), 56.6(\mathrm{C}-17)$, 54.5 (C-5), 51.1 (C-31), 49.6 (C-9), 49.2 (C-18), 47.1 (C-19), 46.9 (C-4), 42.3 (C-8), 40.6 (C-14), 40.5 (C-34), 39.1 (C-1), 38.4 (C-13), 36.6 (C-10), 36.3 (C-22), 33.3 (C-7), 31.4 (C-21), 30.2 (C-16), 29.4 (C-15), 25.8 (C-23), 25.3 (C-12), 21.2 (C-11), 19.9 (C-30), 19.3 (C-6), 18.1 (C-24), 15.2 (C-26), 15.1 (C-25), 13.6 (C-27) ppm; MS (ESI, MeOH): $m / z=702.5\left(100 \%,[\mathrm{M}-\mathrm{Cl}]^{+}\right) ; \mathrm{MS}\left(\mathrm{ESI}, \mathrm{CD}_{3} \mathrm{OD}\right)$ : $m / z=703.7\left(46 \%,[\mathrm{M}+\mathrm{D}]^{+}\right), 704.6(100 \%$, [M$\left.\mathrm{H}+2 \mathrm{D}]^{+}\right), \quad 705.5\left(60 \%, \quad[\mathrm{M}-2 \mathrm{H}+3 \mathrm{D}]^{+}\right) ;$analysis calculated for $\mathrm{C}_{48} \mathrm{H}_{64} \mathrm{ClNO}_{3}$ (738.48): C 78.07, H 8.74, N 1.90; found: C 77.68, H 8.83, N 1.74.

\section{Methyl 3-oxolup-20(29)-en-28-oate 13}

Compound 13 was obtained by esterification of 5 as previously described ${ }^{20}$.

Methyl (3ß) 3-ethynyl-3-hydroxylup-20(29)en-28-oate 14

From 13 (4.69 g, $10.0 \mathrm{mmol})$ and ethynylmagnesium bromide; compound 4 (3.38 g, $68 \%$ ) was obtained as a white solid as previously reported $^{14}$.

Methyl (3ß) 3-[3-(diisopropylamino)prop-1yn-1-yl]-3-hydroxylup-20(29)-en-28-oate hydrochloride 15

Compound 15 was prepared (as described for the synthesis of 24) from $14(297 \mathrm{mg}, 0.6 \mathrm{mmol})$, diisopropylamine $(0.09 \mathrm{~mL}, 0.64 \mathrm{mmol})$, formalin (37\%, $0.25 \mathrm{~mL}, 2.5 \mathrm{mmol})$ and $\mathrm{CuI}(2 \mathrm{mg}, 0.01$ $\mathrm{mmol})$ and $15(155 \mathrm{mg}, 48 \%)$ was obtained as a colorless solid; $\mathrm{mp} 215^{\circ} \mathrm{C} ;[\alpha]_{\mathrm{D}}=-7.7^{\circ}(c=6.15$, $\mathrm{MeOH}) ;{ }^{1} \mathrm{H}$ NMR $\left(500 \mathrm{MHz}, \mathrm{CD}_{3} \mathrm{OD}\right): \delta=4.71$ ( $m, 1 \mathrm{H}, \mathrm{H}-29 \mathrm{a}), 4.60$ ( $m, 1 \mathrm{H}, \mathrm{H}-29 \mathrm{~b}), 4.21$ ( $s, 2 \mathrm{H}, \mathrm{H}-$ 34), 3.90 (sept., $2 \mathrm{H}, J=6.7 \mathrm{~Hz}, \mathrm{H}-35+\mathrm{H}-38), 3.65$ $(s, 3 \mathrm{H}, \mathrm{H}-31), 2.99(d d d, 1 \mathrm{H}, J=10.6,10.6,5.1 \mathrm{~Hz}$, $\mathrm{H}-19), 2.29-2.21$ ( $m, 2 \mathrm{H}, \mathrm{H}-13+\mathrm{H}-16), 1.96(m, 1 \mathrm{H}$, $\mathrm{H}-2 \mathrm{a}), 1.90-1.82$ ( $m, 2 \mathrm{H}, \mathrm{H}-21 \mathrm{a}+\mathrm{H}-22 \mathrm{a}), 1.76-1.65$ $(m, 3 \mathrm{H}, \mathrm{H}-1 \mathrm{a}+\mathrm{H}-12+\mathrm{H}-2 \mathrm{~b}), 1.69(s, 3 \mathrm{H}, \mathrm{H}-30)$, $1.64(d d, 1 \mathrm{H}, J=11.4,11.4 \mathrm{~Hz}, \mathrm{H}-18), 1.54-1.21$ $(m, 12 \mathrm{H}, \mathrm{H}-9+\mathrm{H}-1 \mathrm{~b}+\mathrm{H}-22 \mathrm{~b}+\mathrm{H}-7 \mathrm{a}+\mathrm{H}-7 \mathrm{~b}+$ $\mathrm{H}-16+\mathrm{H}-21 \mathrm{~b}+\mathrm{H}-15 \mathrm{a}+\mathrm{H}-11 \mathrm{a}+\mathrm{H}-11 \mathrm{~b}+\mathrm{H}-6 \mathrm{a}+$ $\mathrm{H}-6 \mathrm{~b}), 1.47(d, 12 \mathrm{H}, J=6.7 \mathrm{~Hz}, \mathrm{C}-36+\mathrm{C}-37+$ C-39 + C-40), 1.20-1.14 ( $m, 2 \mathrm{H}, \mathrm{H}-5+\mathrm{H}-15 \mathrm{~b}), 1.10-$ 1.01 ( $m, 1 \mathrm{H}, \mathrm{H}-12 \mathrm{~b}), 1.05$ ( $s, 3 \mathrm{H}, \mathrm{H}-24), 0.99(s, 3 \mathrm{H}$, H-27), 0.94 ( $s, 3 \mathrm{H}, \mathrm{H}-26), 0.88(s, 3 \mathrm{H}, \mathrm{H}-25), 0.85$ $(s, 3 \mathrm{H}, \mathrm{H}-23) \mathrm{ppm} ;{ }^{13} \mathrm{C}$ NMR (125 MHz, $\left.\mathrm{CD}_{3} \mathrm{OD}\right)$ : $\delta=178.1$ (C-28), 151.8 (C-20), 110.3 (C-29), 94.9 $(\mathrm{C}-32, C \equiv \mathrm{CH}), 76.6(\mathrm{C}-33, \mathrm{C} \equiv \mathrm{CH}), 74.4(\mathrm{C}-3), 57.9$ (C-17), 55.8 (C-35 + C-38), $55.3(\mathrm{C}-5), 52.3(\mathrm{C}-31)$, 51.8 (C-18), 50.7 (C-9), 48.4 (C-19), 43.6 (C-14), 42.9 (C-4), 41.9 (C-8), 39.6 (C-13), 39.2 (C-1), 38.4 (C-10), 37.8 (C-22), 37.1 (C-34), 35.6 (C-7), 33.2 (C-2), 33.1 (C-16), 31.6 (C-21), 30.8 (C-15), 26.9 (C-12), 26.6 (C-24), 22.0 (C-11), 19.6 (C-6), 19.6 (C-30), 18.2 (C-23), $18.2(\mathrm{C}-36+\mathrm{C} 37+\mathrm{C} 39+\mathrm{C} 40)$, 17.0 (C-25), 16.5 (C-26), 15.4 (C-27) ppm; MS (ESI, $\mathrm{MeOH}): m / z=608.5\left(100 \%[\mathrm{M}+\mathrm{H}]^{+}\right) ;$analysis calculated for $\mathrm{C}_{40} \mathrm{H}_{66} \mathrm{ClNO}_{3}(644.41): \mathrm{C}, 74.55 ; \mathrm{H}$, 10.32; N, 2.17; found: C, 74.36; H, 10.56; N, 2.11. 16

\section{(3ß) 3-Hydroxy-lup-20(29)-en-28-yl acetate}

Betulin 1 (10.0 g, $22 \mathrm{mmol}$ ) was acetylated as previously described, and $\mathbf{1 6}(7.8 \mathrm{~g}, 73 \%)$ was obtained as a colorless solid; mp $210-212{ }^{\circ} \mathrm{C}$ (lit.: $\left.210-212{ }^{\circ} \mathrm{C}^{39}\right) ;[\alpha]_{\mathrm{D}}=7.4^{\circ}\left(c=5.7, \mathrm{CHCl}_{3}\right), 8.5^{\circ}(c$ $\left.=1.58, \mathrm{CHCl}_{3}\right)^{39} ; \mathrm{R}_{F}=0.49$ ( $n$-hexane/ethyl acetate, $8 / 2)$.

\section{3-Oxolup-20(29)-en-28-yl acetate 17}

Swern oxidation of $16(10 \mathrm{~g}, 22 \mathrm{mmol})$ as described above followed by an extraction with DCM (4 x $50 \mathrm{~mL})$ and column chromatography $\left(\mathrm{SiO}_{2}\right.$, hexanes/ethyl acetate, 8/2) gave 17 (4.98 g, $81 \%$ ) as a colorless solid; mp 89-91 ${ }^{\circ} \mathrm{C}$ (lit.: 77-79 $\left.{ }^{\circ} \mathrm{C}^{22}\right) ;[\alpha]_{\mathrm{D}}=36.1^{\circ}\left(c=5.1, \mathrm{CHCl}_{3}\right) ; \mathrm{R}_{F}=0.66$ (n-hexane/ethyl acetate, 8/2); ${ }^{1} \mathrm{H}$ NMR (400 MHz, $\left.\mathrm{CDCl}_{3}\right): \delta=4.62(d, 1 \mathrm{H}, J=1.8 \mathrm{~Hz}, \mathrm{H}-29 \mathrm{a}), 4.52$ $(m, 1 \mathrm{H}, \mathrm{H}-29 \mathrm{~b}), 4.19(d d, 1 \mathrm{H}, J=1.5,11.1 \mathrm{~Hz}$, H-28a), 3.79 (d, 1H, $J=10.9 \mathrm{~Hz}, \mathrm{H}-28 \mathrm{~b}), 2.47-2.26$ $(m, 1 \mathrm{H}, \mathrm{H}-19+\mathrm{H}-2), 2.00(s, 3 \mathrm{H}, \mathrm{H}-32), 1.95-1.62$ $(m, 5 \mathrm{H}, \mathrm{H}-21 \mathrm{a}+\mathrm{H}-16+\mathrm{H}-22 \mathrm{a}+\mathrm{H}-1 \mathrm{a}+\mathrm{H}-13), 1.62$ $(s, 3 \mathrm{H}, \mathrm{H}-30), 1.60-1.49(m, 3 \mathrm{H}, \mathrm{H}-18+\mathrm{H}-12+$ $\mathrm{H}-15 \mathrm{a}), 1.43-1.11(m, 11 \mathrm{H}, \mathrm{H}-6+\mathrm{H}-21 \mathrm{~b}+\mathrm{H}-7+$ $\mathrm{H}-11+\mathrm{H}-9+\mathrm{H}-16+\mathrm{H}-1 \mathrm{~b}+\mathrm{H}-5), 1.07-1.01(m, 2 \mathrm{H}$, $\mathrm{H}-15 \mathrm{~b}+\mathrm{H}-12 \mathrm{~b}), 1.00(s, 6 \mathrm{H}, \mathrm{H}-27+\mathrm{H}-25), 0.99(m$, $1 \mathrm{H}, \mathrm{H}-22 \mathrm{~b}), 0.94$ (s, 3H, H-24), 0.92 (s, 3H, H-26), $0.86(s, 3 \mathrm{H}, \mathrm{H}-23) \mathrm{ppm} ;{ }^{13} \mathrm{C} \mathrm{NMR}(125 \mathrm{MHz}$, $\left.\mathrm{CDCl}_{3}\right): \delta=218.1$ (C-3), 171.6 (C-31), 150.0 (C-20), 109.9 (C-29), 62.7 (C-28), 54.9 (C-5), 49.7 (C-9), 48.7 (C-18), 47.6 (C-19), 47.3 (C-4), 46.3 (C-17), 42.7 (C-14), 40.8 (C-8), 39.6 (C-1), 37.6 (C-13), 36.9 (C-10), 34.5 (C-22), 34.1 (C-2), 33.5 (C-7), 29.7 (C-21), 29.6 (C-16), 27.0 (C-12), 26.5 (C-23), 25.2 (C-15), 21.3 (C-11), 21.0 (C-32), 21.0 (C-30), 
19.6 (C-6), 15.9 (C-24 + C-26), 15.8 (C-25), 14.7 (C-27) ppm; MS (ESI, MeOH): $m / z=483.3(32 \%$, $\left.[\mathrm{M}+\mathrm{H}]^{+}\right), \quad 537.1\left(49 \%,[\mathrm{M}+\mathrm{Na}+\mathrm{MeOH}]^{+}\right), \quad 746.5$ $\left(100 \%,[3 \mathrm{M}+2 \mathrm{Na}]^{2+}\right), 987.3\left(67 \%,[2 \mathrm{M}+\mathrm{Na}]^{+}\right)$; analysis calculated for $\mathrm{C}_{32} \mathrm{H}_{50} \mathrm{O}_{3}$ (482.74): $\mathrm{C} 79.62$, H 10.64; found: C 79.55, H 10.79 .

\section{(3ß) 3-Ethynyl-3-hydroxy-lup-20(29)-en-28- yl acetate 18}

As described for the synthesis of $\mathbf{1 4}$, reaction of $17(4.2 \mathrm{~g}, 8.7 \mathrm{mmol})$ with ethinylmagnesium bromide $(0.5 \mathrm{M}, 52 \mathrm{~mL}, 26 \mathrm{mmol})$ followed by chromatographic workup (column, $\mathrm{SiO}_{2}$, hexanes/ethyl acetate, 8/2) yielded $\mathbf{1 8}(3.35 \mathrm{~g}, 75 \%)$ as a colorless solid; mp $109-110{ }^{\circ} \mathrm{C} ;[\alpha]_{\mathrm{D}}=14.3^{\circ}(c$ $\left.=3.8, \mathrm{CHCl}_{3}\right) ; \mathrm{R}_{F}=0.55$ (n-hexane/ethyl acetate, 8/2); ${ }^{1} \mathrm{H}$ NMR $\left(400 \mathrm{MHz}, \mathrm{CDCl}_{3}\right): \delta=4.66(d, 1 \mathrm{H}$, $J=1.6 \mathrm{~Hz}, \mathrm{H}-29 \mathrm{a}), 4.56(d, 1 \mathrm{H}, J=1.2 \mathrm{~Hz}, \mathrm{H}-29 \mathrm{~b})$, $4.23(d, 1 \mathrm{H}, J=10.9 \mathrm{~Hz}, \mathrm{H}-28 \mathrm{a}), 3.84(d, 1 \mathrm{H}, J=$ $11.0 \mathrm{~Hz}, \mathrm{H}-28 \mathrm{~b}), 2.47-2.36$ ( $m, 2 \mathrm{H}, \mathrm{H}-19+\mathrm{H}-34)$, 2.04 ( $s, 3 \mathrm{H}, \mathrm{H}-32), 2.00-1.85$ ( $m, 2 \mathrm{H}, \mathrm{H}-21 \mathrm{a}+\mathrm{H}-2 \mathrm{a})$, 1.85-1.79 ( $m, 1 \mathrm{H}, \mathrm{H}-16), 1.74(d d, 1 \mathrm{H}, J=8.4,12.4$ $\mathrm{Hz}, \mathrm{H}-22 \mathrm{a}), 1.71-1.67$ ( $m, 1 \mathrm{H}, \mathrm{H}-1 \mathrm{a}), 1.65(s, 3 \mathrm{H}$, $\mathrm{H}-30), 1.64-1.52(m, 5 \mathrm{H}, \mathrm{H}-18+\mathrm{H}-2 \mathrm{~b}+\mathrm{H}-13+$ $\mathrm{H}-12+\mathrm{H}-15 \mathrm{a}), 1.51-1.20(m, 10 \mathrm{H}, \mathrm{H}-6+\mathrm{H}-21 \mathrm{~b}+$ $\mathrm{H}-1 \mathrm{~b}+\mathrm{H}-7+\mathrm{H}-11 \mathrm{a}+\mathrm{H}-12 \mathrm{~b}+\mathrm{H}-9+\mathrm{H}-16), 1.19-$ $1.04(m, 4 \mathrm{H}, \mathrm{H}-22 \mathrm{~b}+\mathrm{H}-15 \mathrm{~b}+\mathrm{H}-11 \mathrm{~b}+\mathrm{H}-5), 1.03$ $(s, 3 \mathrm{H}, \mathrm{H}-27), 1.01$ ( $s, 3 \mathrm{H}, \mathrm{H}-25), 0.97$ ( $s, 3 \mathrm{H}, \mathrm{H}-24)$, $0.84(s, 3 \mathrm{H}, \mathrm{H}-26), 0.81(m, 3 \mathrm{H}, \mathrm{H}-23) \mathrm{ppm} ;{ }^{13} \mathrm{C}$ NMR $\left(125 \mathrm{MHz}, \mathrm{CDCl}_{3}\right): \delta=171.6(\mathrm{C}-31), 150.1$ (C-20), 109.8 (C-29), $87.2(\mathrm{C}-33, C \equiv \mathrm{CH}), 75.7$ (C3), $73.5(\mathrm{C}-34, \mathrm{C} \equiv C \mathrm{H}), 62.8(\mathrm{C}-28), 53.1(\mathrm{C}-5), 50.4$ (C-9), 48.8 (C-18), 47.7 (C-19), 46.3 (C-17), 42.7 (C-14), 41.3 (C-1), 40.9 (C-8), 38.9 (C-4), 37.6 (C-13), 37.1 (C-10), 34.5 (C-7), 34.2 (C-22), 29.8 (C-21), 29.6 (C-16), 27.2 (C-12), 25.6 (C-23), 25.6 (C-15), 25.2 (C-2), 21.0 (C-32), 20.8 (C-11), 19.1 (C-30), 18.5 (C-6), 17.4 (C-24), 16.4 (C-26), 16.0 (C-25, $\mathrm{CH}_{3}, 14.9$ (C-27) ppm; MS (ESI, $\left.\mathrm{MeOH}\right): \mathrm{m} / \mathrm{z}$ $=531.5\left(22 \%,[\mathrm{M}+\mathrm{Na}]^{+}\right), \quad 563.1 \quad(34 \%,[\mathrm{M}+$ $\left.\mathrm{MeOH}]^{+}\right), \quad 1039.3\left(100 \%,[2 \mathrm{M}+\mathrm{H}]^{+}\right) ;$analysis calculated for $\mathrm{C}_{34} \mathrm{H}_{52} \mathrm{O}_{3}$ (508.77): C 80.26, H 10.30; found: C 80.02, H 10.41.

(3ß) 3-[3-(Diisopropylamino)-prop-1-yn-1yl]-3-hydroxylup-20(29)-en-28-yl acetate 19

Following the procedure given for $\mathbf{8}$, from the reaction of 18 (500 mg, $1 \mathrm{mmol})$, diisopropylamine (160 $\mu \mathrm{L}, 1.15 \mathrm{mmol})$, formalin $(37 \%, 0.4 \mathrm{~mL}, 5$ $\mathrm{mmol})$ and $\mathrm{CuI}(20 \mathrm{mg}, 0.1 \mathrm{mmol})$ followed by chromatographic workup (column, $\mathrm{SiO}_{2}$, hexanes/ethyl acetate, 8/2) 19 (464 mg, 74\%) was obtained as a colorless solid; $\mathrm{mp} 132-135{ }^{\circ} \mathrm{C} ;[\alpha]_{\mathrm{D}}=$ $7.3^{\circ}\left(c=3.7, \mathrm{CHCl}_{3}\right) ; \mathrm{R}_{F}=0.04$ (n-hexane/ethyl acetate, 8/2); ${ }^{1} \mathrm{H}$ NMR (400 MHz, $\left.\mathrm{CDCl}_{3}\right): \delta=4.63$ $(d, 1 \mathrm{H}, J=1.7 \mathrm{~Hz}, \mathrm{H}-29 \mathrm{a}), 4.55-4.51(m, 1 \mathrm{H}$, $\mathrm{H}-29 \mathrm{~b}), 4.19$ ( $d, 1 \mathrm{H}, J=10.4 \mathrm{~Hz}, \mathrm{H}-28 \mathrm{a}), 3.91-3.85$ ( $s$ br, 1H, H-33), $3.78(d, 1 \mathrm{H}, J=11.1 \mathrm{~Hz}, \mathrm{H}-28 \mathrm{~b})$, 3.68-3.50 ( $m, 2 \mathrm{H}, \mathrm{H}-34), 2.38(d d d, 1 \mathrm{H}, J=5.9$, $11.1,11.2 \mathrm{~Hz}, \mathrm{H}-19), 2.00\left(s, 3 \mathrm{H}, \mathrm{CH}_{3}(37)\right), 1.95-$ $1.63(m, 6 \mathrm{H}, \mathrm{H}-21 \mathrm{a}+\mathrm{H}-16+\mathrm{H}-22 \mathrm{a}+\mathrm{H}-15 \mathrm{a}+\mathrm{H}-13$
$+\mathrm{H}-2 \mathrm{a}), 1.62(s, 3 \mathrm{H}, \mathrm{H}-30), 1.61-1.48(m, 4 \mathrm{H}, \mathrm{H}-1 \mathrm{a}$ $+\mathrm{H}-2 \mathrm{~b}+\mathrm{H}-18+\mathrm{H}-12), 1.46-0.98(m, 26 \mathrm{H}, \mathrm{H}-35+$ $\mathrm{H}-6+\mathrm{H}-21 \mathrm{~b}+\mathrm{H}-1 \mathrm{~b}+\mathrm{H}-7+\mathrm{H}-11+\mathrm{H}-15 \mathrm{~b}+\mathrm{H}-5+$ $\mathrm{H}-12 \mathrm{~b}+\mathrm{H}-22 \mathrm{~b}+\mathrm{H}-9+\mathrm{H}-16), 0.96(s, 3 \mathrm{H}, \mathrm{H}-27)$, $0.95(s, 3 \mathrm{H}, \mathrm{H}-25), 0.89$ ( $s, 3 \mathrm{H}, \mathrm{H}-24), 0.80(s, 3 \mathrm{H}$, $\mathrm{H}-26), 0.77$ ( $s, 3 \mathrm{H}, \mathrm{H}-23) \mathrm{ppm} ;{ }^{13} \mathrm{C} \mathrm{NMR}(125 \mathrm{MHz}$, $\left.\mathrm{CDCl}_{3}\right): \delta=171.6(\mathrm{C}-36), 150.1(\mathrm{C}-20), 109.9$ (C-29), 90.4 (C-31, $C \equiv \mathrm{C}), 76.0$ (C-3), 75.0 (C-32, $\mathrm{C} \equiv C), 62.7$ (C-28), 53.9 (C-5 + C-34), 50.8 (C-9), 48.8 (C-18), 47.7 (C-19), 46.3 (C-17), 42.7 (C-14), 41.7 (C-4), 40.9 (C-8), 38.1 (C-1), 37.6 (C-13), 37.2 (C-10), $34.5\left(\mathrm{C}-22+\mathrm{C} 33,2 \times \mathrm{CH}_{2}\right), 34.3(\mathrm{C}-7), 32.6$ (C-2), 29.7 (C-21), 29.6 (C-16), 27.0 (C-15), 26.0 (C-23), 25.2 (C-12), 21.0 (C-37), 20.8 (C-11), 19.2 $(\mathrm{C}-30+\mathrm{C}-35), 18.5$ (C-6), 17.5 (C-24), 16.4 (C-26), 16.0 (C-25), 14.9 (C-27) ppm; MS (ESI, MeOH): $m / z=622.6\left(100 \%,[\mathrm{M}+\mathrm{H}]^{+}\right)$; analysis calculated for $\mathrm{C}_{41} \mathrm{H}_{67} \mathrm{NO}_{3}$ (621.98): C 79.12, H 10.86; found: C 78.98, H 10.92, N 10.61 .

\section{Methyl (3ß) 3-hydroxy-11-oxo-olean-12-en- 30-oate 20}

Esterification of glycyrrhetinic acid $(4,5.0 \mathrm{~g}$, $10.6 \mathrm{mmol})$ in DMF $(50 \mathrm{~mL})$ with potassium carbonate $(2.49 \mathrm{~g}, 18 \mathrm{mmol})$ and methyl iodide $(806$ $\mu \mathrm{L}, 12.9 \mathrm{mmol}$ ) as previously described followed by chromatographic workup (column, $\mathrm{SiO}_{2}$, hexanes/ethyl acetate, 8/2) furnished 20 (4.98 g, 97\%) as a colorless solid; mp $254-256{ }^{\circ} \mathrm{C}$ (lit.: 254$\left.258^{\circ} \mathrm{C}^{7}\right) ;[\alpha]_{\mathrm{D}}=141.4^{\circ}\left(c=0.32, \mathrm{CHCl}_{3}\right) ; \mathrm{R}_{F}=0.27$ (n-hexane/ethyl acetate, $8 / 2$ ).

\section{Benzyl (3ß) 3-hydroxy-11-oxo-olean-12-en- 30-oate 21}

Reaction of $4(5.0 \mathrm{~g}, 10.6 \mathrm{mmol})$ in DMF (75 $\mathrm{mL})$ with potassium carbonate $(2.49 \mathrm{~g}, 18 \mathrm{mmol})$ and benzyl bromide $(1.5 \mathrm{~mL}, 12.9 \mathrm{mmol})$ followed by chromatographic workup (column, $\mathrm{SiO}_{2}$, hexanes/ ethyl acetate, 8/2) gave $\mathbf{2 1}^{24}(4.46 \mathrm{~g}, 75 \%)$ as a colorless solid; mp $137-139{ }^{\circ} \mathrm{C} ;[\alpha]_{\mathrm{D}}=137.4^{\circ}(c=$ $\left.0.20, \mathrm{CHCl}_{3}\right) ; \mathrm{R}_{F}=0.45$ ( $n$-hexane/ethyl acetate, $4: 1) ;{ }^{1} \mathrm{H}$ NMR (500 MHz, $\left.\mathrm{CDCl}_{3}\right): \delta=7.33-7.23$ ( $m, 5 \mathrm{H}$, phenyl), $5.48(s, 1 \mathrm{H}, \mathrm{H}-12), 5.13(d, 1 \mathrm{H}$, $J=12.2 \mathrm{~Hz}, \mathrm{H}-31 \mathrm{a}), 5.02(d, 1 \mathrm{H}, J=12.2 \mathrm{~Hz}, \mathrm{H}-$ $31 \mathrm{~b}), 3.15$ ( $d d, 1 \mathrm{H}, J=5.5,10.8 \mathrm{~Hz}, \mathrm{H}-3), 2.72$ (ddd, $1 \mathrm{H}, J=3.5,3.5,13.4 \mathrm{~Hz}, \mathrm{H}-1 \mathrm{a}), 2.25(s, 1 \mathrm{H}, \mathrm{H}-9)$, 2.00-1.89 ( $m, 3 \mathrm{H}, \mathrm{H}-18+\mathrm{H}-21 \mathrm{a}+\mathrm{H}-16), 1.86$ (ddd, $1 \mathrm{H}, J=2.7,4.1,13.6 \mathrm{~Hz}, \mathrm{H}-19 \mathrm{a}), 1.78-1.69(m, 1 \mathrm{H}$, $\mathrm{H}-15 \mathrm{a}), 1.65-1.48\left(m, 5 \mathrm{H}, \mathrm{CH}_{2}(2)+\mathrm{H}-22 \mathrm{a}+\mathrm{H}-19 \mathrm{~b}\right.$ $+\mathrm{H}-6 \mathrm{a}), 1.47-1.17(m, 8 \mathrm{H}, \mathrm{H}-7+\mathrm{H}-21 \mathrm{~b}+\mathrm{H}-22 \mathrm{~b}+$ $\mathrm{H}-6 \mathrm{~b}+\mathrm{H}-27), 1.13-1.08$ ( $m, 4 \mathrm{H}, \mathrm{H}-28+\mathrm{H}-16), 1.06$ $(s, 3 \mathrm{H}, \mathrm{H}-26), 1.04(s, 3 \mathrm{H}, \mathrm{H}-25), 0.96-0.85(m, 5 \mathrm{H}$, $\mathrm{H}-1 \mathrm{~b}+\mathrm{H}-23+\mathrm{H}-15 \mathrm{~b}), 0.73(s, 3 \mathrm{H}, \mathrm{H}-24), 0.66(s$, $3 \mathrm{H}, \mathrm{H}-29), 0.62(d, 1 \mathrm{H}, J=10.1 \mathrm{~Hz}, \mathrm{H}-5) \mathrm{ppm} ;{ }^{13} \mathrm{C}$ NMR $\left(100 \mathrm{MHz}, \mathrm{CDCl}_{3}\right): \delta=200.1(\mathrm{C}-11), 176.2$ (C-30), $168.9(\mathrm{C}-13, C=\mathrm{CH}), 136.1(\mathrm{Ph}), 128.6(\mathrm{C}-$ $12, \mathrm{C}=C \mathrm{H}), 128.6(\mathrm{Ph}), 128.5(\mathrm{Ph}), 128.3(\mathrm{Ph}), 128.2$ $(\mathrm{Ph}), 128.2(\mathrm{Ph}), 78.7(\mathrm{C}-3), 66.2(\mathrm{C}-31), 61.8(\mathrm{C}-9)$, 54.9 (C-5), 48.2 (C-18), 45.3 (C-14), 43.9 (C-20), 43.1 (C-8), 41.1 (C-19), 39.1 (C-1), 39.1 (C-4), 37.6 (C-22), 37.0 (C-10), 32.7 (C-7), 31.7(C-17), 31.1 
(C-21), 28.4 (C-29), 27.3 (C-28), 28.1 (C-23), 27.3 (C-2), 26.4 (C-15 + C16), 23.6 (C-27), 18.6 (C-26), 17.5 (C-6), 16.3 (C-25), 15.5 (C-24) ppm; MS (ESI, $\mathrm{MeOH}): m / z=561.5\left(56 \%,[\mathrm{M}+\mathrm{H}]^{+}\right), 583.4(26 \%$, $\left.[\mathrm{M}+\mathrm{Na}]^{+}\right), 863.9\left(10 \%,[3 \mathrm{M}+2 \mathrm{Na}]^{2+}\right), 1121.2(54 \%$, $\left.[2 \mathrm{M}+\mathrm{H}]^{+}\right), 1143.3\left(100 \%,[2 \mathrm{M}+\mathrm{Na}]^{+}\right)$; analysis calculated for $\mathrm{C}_{37} \mathrm{H}_{52} \mathrm{O}_{4}$ (560.81): $\mathrm{C} 79.24$, H 9.35; found: $79.03, \mathrm{H} 9.51$.

Methyl 3,11-dioxo-olean-12-en-30-oate 22

Swern oxidation of $\mathbf{2 0}$ (4.98 g, $10.1 \mathrm{mmol})$ with DMSO (1.4 mL, $20 \mathrm{mmol})$, oxalyl chloride $(1.7 \mathrm{~mL}$, $20 \mathrm{mmol})$ and triethylamine $(42 \mathrm{~mL}, 0.3 \mathrm{~mol}$ ) as described above followed by chromatographic workup (column, $\mathrm{SiO}_{2}$, hexanes/ethyl acetate, 8/2) gave 22 (3.95 g, 87\%) as a colorless solid; mp 242$243{ }^{\circ} \mathrm{C} ;[\alpha]_{\mathrm{D}}=187.7^{\circ}\left(c=0.61, \mathrm{CHCl}_{3}\right) ; \mathrm{R}_{F}=0.50$ (n-hexane/ethyl acetate, $8 / 2) .^{25}$

\section{Benzyl 3,11-dioxo-olean-12-en-30-oate 23}

Jones oxidation of $21(2.0 \mathrm{~g}, 3.5 \mathrm{mmol})$ as described above followed by extraction with DCM (Soxhlet apparatus, $12 \mathrm{~h}$ ) and column chromatography $\left(\mathrm{SiO}_{2}\right.$, hexanes/ethyl acetate, 8/2) gave $23{ }^{25}(1.57 \mathrm{~g}, 87 \%)$ as a colorless solid; $\mathrm{mp}$ 136$138{ }^{\circ} \mathrm{C} ;[\alpha]_{\mathrm{D}}=185.5^{\circ}\left(c=4.45, \mathrm{CHCl}_{3}\right) ; \mathrm{R}_{F}=0.25$ (n-hexane/ethyl acetate, 4/1); ${ }^{1} \mathrm{H}$ NMR $(500 \mathrm{MHz}$, $\left.\mathrm{CDCl}_{3}\right): \delta=7.34-7.23$ ( $m, 5 \mathrm{H}$, aromat), $5.51(s, 1 \mathrm{H}$, $\mathrm{H}-12), 5.14(d, 1 \mathrm{H}, J=12.2 \mathrm{~Hz}, \mathrm{H}-31 \mathrm{a}), 5.02(d, 1 \mathrm{H}$, $J=12.2 \mathrm{~Hz}, \mathrm{H}-31 \mathrm{~b}), 2.89$ (ddd, 1H, $J=4.1,7.1,13.6$ $\mathrm{Hz}, \mathrm{H}-1 \mathrm{a}), 2.56$ (ddd, $1 \mathrm{H}, J=7.1,11.2,15.8 \mathrm{~Hz}$, $\mathrm{H}-2 \mathrm{a}), 2.35$ ( $s, 1 \mathrm{H}, \mathrm{H}-9), 2.29$ ( $d d d, 1 \mathrm{H}, J=4.0,6.5$, $15.8 \mathrm{~Hz}, \mathrm{H}-2 \mathrm{~b}), 2.00$ (dd, 1H, $J=3.9,9.0 \mathrm{~Hz}, \mathrm{H}-18)$, 1.97-1.90 ( $m, 2 \mathrm{H}, \mathrm{H}-21 \mathrm{a}+\mathrm{H}-16), 1.87$ (ddd, 1H, $J=2.8,4.1,13.6 \mathrm{~Hz}, \mathrm{H}-19 \mathrm{a}), 1.81-1.71(m, 1 \mathrm{H}$, $\mathrm{H}-15 \mathrm{a}), 1.65-1.43(m, 4 \mathrm{H}, \mathrm{H}-22 \mathrm{a}+\mathrm{H}-19 \mathrm{~b}+\mathrm{H}-6)$, $1.41-1.16(m, 12 \mathrm{H}, \mathrm{H}-5+\mathrm{H}-1 \mathrm{~b}+\mathrm{H}-7+\mathrm{H}-21 \mathrm{~b}+\mathrm{H}-$ $28+\mathrm{H}-22 \mathrm{~b}+\mathrm{H}-27), 1.12-1.06(m, 7 \mathrm{H}, \mathrm{H}-26+\mathrm{H}-25$ $+\mathrm{H}-16), 1.03$ ( $s, 3 \mathrm{H}, \mathrm{H}-23), 1.00$ ( $s, 3 \mathrm{H}, \mathrm{H}-24), 0.97-$ $0.90(m, 1 \mathrm{H}, \mathrm{H}-15 \mathrm{~b}), 0.68(s, 3 \mathrm{H}, \mathrm{H}-29) \mathrm{ppm} ;{ }^{13} \mathrm{C}$ NMR (125 MHz, $\left.\mathrm{CDCl}_{3}\right): \delta=217.1$ (C-3), 199.3 (C-11), 176.1 (C-30), 169.4 (C-13), $136.1(\mathrm{Ph})$, $128.6(\mathrm{C}-12), 128.4(\mathrm{Ph}), 128.3(\mathrm{Ph}), 128.3(\mathrm{Ph})$, $128.2(\mathrm{Ph}), 128.2(\mathrm{Ph}), 66.2(\mathrm{C}-31), 61.0(\mathrm{C}-9), 55.4$ (C-5), 48.2 (C-18), 47.8 (C-4), 45.2 (C-14), 43.9 (C-20), 43.3 (C-8), 41.1 (C-19), 39.8 (C-1), 37.6 (C-22), 36.7 (C-10), 34.2 (C-2), 32.1 (C-7), 31.8 (C-17), 31.1 (C-21), 28.4 (C-29), 28.3 (C-28), 26.4 (C-15 + C-16), 26.4 (C-23), 23.3 (C-27), 21.4 (C-24), 18.8 (C-6), 18.5 (C-26), 15.6 (C-25) ppm; MS (ESI, $\mathrm{MeOH}): m / z=559.6\left(76 \%,[\mathrm{M}+\mathrm{H}]^{+}\right)$, $581.5\left(20 \%,[\mathrm{M}+\mathrm{Na}]^{+}\right), 866.9\left(50 \%,[3 \mathrm{M}+2 \mathrm{Na}]^{2+}\right)$, $1117.3\left(72 \%,[2 \mathrm{M}+\mathrm{H}]^{+}\right), 1139.2\left(100 \%,[2 \mathrm{M}+\mathrm{Na}]^{+}\right)$; analysis calculated for $\mathrm{C}_{37} \mathrm{H}_{50} \mathrm{O}_{4}$ (558.79): $\mathrm{C} 79.53$, H 9.02; found: C 79.41, H 9.17.

\section{Methyl (3ß) 3-ethynyl-3-hydroxy-11-oxo- olean-12-en-30-oate 24}

Reaction of 22 (500 mg, $1.03 \mathrm{mmol}$ ) in anhydrous THF $(30 \mathrm{~mL})$ with ethynylmagnesium bromide $(0.5 \mathrm{M}$ in THF, $4 \mathrm{~mL}, 2 \mathrm{mmol})$ at $25^{\circ} \mathrm{C}$ for
72 hours followed by chromatography $\left(\mathrm{SiO}_{2}\right.$, hexanes/ethyl acetate, 8/2) yielded 24 (450 mg, $86 \%$ ) as a colorless solid; $\mathrm{mp} 231-235^{\circ} \mathrm{C}$ (lit.: 247$\left.247.5{ }^{\circ} \mathrm{C}^{26}\right) ;[\alpha]_{\mathrm{D}}=112.6^{\circ}\left(c=3.1, \mathrm{CHCl}_{3}\right)$; $\mathrm{R}_{F}=0.27$ ( $n$-hexane/ethyl acetate, 8/2); ${ }^{1} \mathrm{H}$ NMR $\left(500 \mathrm{MHz}, \mathrm{CDCl}_{3}\right): \delta=5.66(s, 1 \mathrm{H}, \mathrm{H}-12), 3.68(s$, $\left.3 \mathrm{H}, \mathrm{CH}_{3}(31)\right), 3.00-2.78$ (ddd, $1 \mathrm{H}, J=3.4,3.4,13.8$ Hz, H-1a), 2.45 ( $s, 1 \mathrm{H}, \mathrm{H}-33$ ), 2.42 ( $s, 1 \mathrm{H}, \mathrm{H}-9), 2.08$ $(d d, 1 \mathrm{H}, J=3.8,14.1 \mathrm{~Hz}, \mathrm{H}-18), 2.05-1.97(m, 3 \mathrm{H}$, $\mathrm{H}-21 \mathrm{a}+\mathrm{H}-2 \mathrm{a}+\mathrm{H}-16), 1.90(d d d, 1 \mathrm{H}, J=2.8,4.1$, $13.6 \mathrm{~Hz}, \mathrm{H}-19 \mathrm{a}), 1.83$ ( $m, 1 \mathrm{H}, \mathrm{H}-15 \mathrm{a}), 1.73-1.53$ ( $m$, $4 \mathrm{H}, \mathrm{H}-7 \mathrm{a}+\mathrm{H}-2 \mathrm{~b}+\mathrm{H}-19 \mathrm{~b}+\mathrm{H}-6 \mathrm{a}), 1.47-1.28(m, 9 \mathrm{H}$, $\mathrm{H}-27+\mathrm{H}-6 \mathrm{~b}+\mathrm{H}-7 \mathrm{~b}+\mathrm{H}-16+\mathrm{H}-1 \mathrm{~b}+\mathrm{H}-22), 1.22-$ $1.15(m, 2 \mathrm{H}, \mathrm{H}-21 \mathrm{~b}+\mathrm{H}-5), 1.14(s, 6 \mathrm{H}, \mathrm{H}-28+\mathrm{H}-$ 25), 1.12 ( $s, 3 \mathrm{H}, \mathrm{H}-26), 1.08$ ( $s, 3 \mathrm{H}, \mathrm{H}-23), 1.04-0.98$ $(m, 1 \mathrm{H}, \mathrm{H}-15 \mathrm{~b}), 0.90(s, 3 \mathrm{H}, \mathrm{H}-24), 0.80(s, 3 \mathrm{H}$, $\mathrm{H}-29) \mathrm{ppm} ;{ }^{13} \mathrm{C}$ NMR $\left(125 \mathrm{MHz}, \mathrm{CDCl}_{3}\right): \delta=200.1$ (C-11), 176.9 (C-30), $169.1(\mathrm{C}-13, C=\mathrm{CH}), 128.4$ (C-12), $87.0(\mathrm{C}-32, C \equiv \mathrm{CH}), 75.4(\mathrm{C}-33, \mathrm{C} \equiv \mathrm{CH})$, 73.5 (C-3), 61.9 (C-9), 52.5 (C-5), 51.7 (C-31), 48.3 (C-18), 45.3 (C-14), 44.0 (C-20), 43.1 (C-8), 41.3 (C-19), 41.1 (C-4), 38.1 (C-1), 37.7 (C-22), 37.0 (C-10), 32.6 (C-7), 32.4 (C-2), 31.8 (C-21), 31.1 (C-17), 28.5 (C-29), 28.2 (C-28), 26.4 (C-15 + C-16), 25.7 (C-23), 23.4 (C-27), 18.6 (C-26), 17.6 (C-6), 17.6 (C-25), 16.7 (C-24) ppm; MS (ESI, $\mathrm{MeOH}): \mathrm{m} / z=509.6\left(100 \%,[\mathrm{M}+\mathrm{H}]^{+}\right), 1017.3(94 \%$, $\left.[2 \mathrm{M}+\mathrm{H}]^{+}\right), \quad 1039.3\left(51 \%,[2 \mathrm{M}+\mathrm{Na}]^{+}\right) ;$analysis calculated for $\mathrm{C}_{33} \mathrm{H}_{48} \mathrm{O}_{4}$ (508.73): C 77.91, H 9.51; found: C 77.85, H 9.63.

\section{Benzyl (3ß)-3-ethynyl-3-hydroxy-11-oxo- olean-12-en-30-oate 25}

From the reaction of $\mathbf{2 3}(2.0 \mathrm{~g}, 4 \mathrm{mmol})$ with ethynylmagnesium bromide $(0.5 \mathrm{M}$ in THF, $20 \mathrm{~mL}$, $10 \mathrm{mmol}$ ) as described above followed by chromatographic workup (column, $\mathrm{SiO}_{2}$, hexanes/ethyl acetate, 8/2) gave 25 (1.58 g, 75\%) as a colorless solid; mp 176-178 ${ }^{\circ} \mathrm{C} ;[\alpha]_{\mathrm{D}}=1200^{\circ}$ $\left(c=0.56, \mathrm{CHCl}_{3}\right) ; \mathrm{R}_{F}=0.33$ ( $n$-hexane/ethyl acetate, 8/2); ${ }^{1} \mathrm{H}$ NMR (500 MHz, $\left.\mathrm{CDCl}_{3}\right): \delta=7.34-7.23(\mathrm{~m}$, $5 \mathrm{H}$, aromat), $5.49(s, 1 \mathrm{H}, \mathrm{H}-12), 5.13(d, 1 \mathrm{H}, J=12.3$ Hz, H-31a), 5.02 (d, 1H, $J=12.3 \mathrm{~Hz}, \mathrm{H}-31 \mathrm{~b}), 2.71$ $(d d d, 1 \mathrm{H}, J=3.5,3.5,13.8 \mathrm{~Hz}, \mathrm{H}-1 \mathrm{a}), 2.38(s, 1 \mathrm{H}$, H-33), 2.33 ( $s, 1 \mathrm{H}, \mathrm{H}-9), 2.08-1.90$ ( $m, 4 \mathrm{H}, \mathrm{H}-18+$ $\mathrm{H}-21 \mathrm{a}+\mathrm{H}-2 \mathrm{a}+\mathrm{H}-15 \mathrm{a}), 1.86(d d d, 1 \mathrm{H}, J=2.8,4.3$, 13.7 Hz, H-19a), 1.79-1.69 (m, 1H, H-16), 1.66-1.43 $(m, 4 \mathrm{H}, \mathrm{H}-7 \mathrm{a}+\mathrm{H}-2 \mathrm{~b}+\mathrm{H}-19 \mathrm{~b}+\mathrm{H}-6 \mathrm{a}), 1.41-1.32$ $(m, 2 \mathrm{H}, \mathrm{H}-6 \mathrm{~b}+\mathrm{H}-1 \mathrm{~b}), 1.31(s, 3 \mathrm{H}, \mathrm{H}-27), 1.30-1.16$ $(m, 4 \mathrm{H}, \mathrm{H}-21 \mathrm{~b}+\mathrm{H}-7 \mathrm{~b}+\mathrm{H}-22), 1.13-1.05(m, 8 \mathrm{H}$, $\mathrm{H}-28+\mathrm{H}-25+\mathrm{H}-16+\mathrm{H}-5), 1.04(s, 3 \mathrm{H}, \mathrm{H}-26), 1.01$ $(s, 3 \mathrm{H}, \mathrm{H}-23), 0.96-0.89$ ( $m, 1 \mathrm{H}, \mathrm{H}-15 \mathrm{~b}), 0.81(s, 3 \mathrm{H}$, $\mathrm{H}-24), 0.67$ ( $s, 3 \mathrm{H}, \mathrm{H}-29) \mathrm{ppm} ;{ }^{13} \mathrm{C}$ NMR $(125 \mathrm{MHz}$, $\left.\mathrm{CDCl}_{3}\right): \delta=200.1$ (C-11), 176.2 (C-30), 169.0 (C-13), 136.1 (Ph), 128.6 (Ph), 128.5 (C-12), 128.3 $(\mathrm{Ph}), 128.2(\mathrm{Ph}), 87.1(\mathrm{C}-32, C \equiv \mathrm{CH}), 75.4(\mathrm{C}-33$, $\mathrm{C} \equiv C \mathrm{H}), 73.5$ (C-3), 66.2 (C-31), 61.9 (C-9), 52.6 (C-5), 48.2 (C-18), 45.3 (C-14), 44.0 (C-20), 43.1 (C-8), 41.4 (C-19), 41.1 (C-4), 38.1 (C-1), 37.7 (C-22), 37.0 (C-10), 32.7 (C-7), 32.5 (C-2), 31.8 (C-21), 31.2 (C-17), 28.4 (C-29), 28.3 (C-28), 26.5 
(C-15), 26.4 (C-16), 25.8 (C-23), 23.4 (C-27), 18.7 (C-26), 17.7 (C-6), 17.6 (C-25), 16.8 (C-24) ppm; MS (ESI, MeOH): $m / z=585.5\left(38 \%,[\mathrm{M}+\mathrm{H}]^{+}\right)$, $607.4\left(8 \%,[\mathrm{M}+\mathrm{Na}]^{+}\right), 1169.4\left(94 \%,[2 \mathrm{M}+\mathrm{H}]^{+}\right)$, $1191.1\left(100 \%,[2 \mathrm{M}+\mathrm{Na}]^{+}\right)$; analysis calculated for $\mathrm{C}_{39} \mathrm{H}_{52} \mathrm{O}_{4}$ (584.83x): C 80.09, H 8.96; found: C 79.77, H 9.04.

\section{Methyl (3ß) 3-[3-(diallylamino)-prop-1-yn-1- yl]- 3-hydroxy-11-oxo-olean-12-en-30-oate hydrochloride 26}

Mannich reaction of $\mathbf{2 4}(1 \mathrm{~g}, 2 \mathrm{mmol})$ in DMSO (7 mL) with diallylamine $(284 \mu \mathrm{L}, 2.3 \mathrm{mmol})$, formalin $(37 \%, 0.8 \mathrm{~mL}, 10 \mathrm{mmol})$ and $\mathrm{CuI}(20 \mathrm{mg}$, $0.1 \mathrm{mmol}$ ) as described above followed by chromatographic workup (column, $\mathrm{SiO}_{2}$, hexanes/ ethyl acetate, 8/2) gave $26(948 \mathrm{mg}, 81 \%)$ as a colorless solid; mp $244-247^{\circ} \mathrm{C} ;[\alpha]_{\mathrm{D}}=742^{\circ}(c=6.0$, $\mathrm{CHCl}_{3}$ ); $\mathrm{R}_{F}=0.05$ ( $n$-hexane/ethyl acetate, $\left.8 / 2\right) ;{ }^{1} \mathrm{H}$ NMR (500 MHz, $\left.\mathrm{CDCl}_{3}\right): \delta=6.03-5.92(m, 2 \mathrm{H}$, $\mathrm{H}-36), 5.67$ ( $s, 1 \mathrm{H}, \mathrm{H}-12), 5.59-5.49$ ( $m, 4 \mathrm{H}, \mathrm{H}-37)$, 3.93 ( $s, 2 \mathrm{H}, \mathrm{H}-34), 3.73-3.64$ ( $m, 7 \mathrm{H}, \mathrm{H}-31+\mathrm{H}-35)$, 2.92-2.84 ( $m, 1 \mathrm{H}, \mathrm{H}-1 \mathrm{a}), 2.34$ ( $s, 1 \mathrm{H}, \mathrm{H}-9), 2.12-1.96$ $(m, 4 \mathrm{H}, \mathrm{H}-18+\mathrm{H}-21 \mathrm{a}+\mathrm{H}-2 \mathrm{a}+\mathrm{H}-16), 1.93-1.86(m$, 1H, H-19a), 1.82 (ddd, 1H, $J=4.3,13.6,13.6 \mathrm{~Hz}$, $\mathrm{H}-15 \mathrm{a}), 1.74-1.35(m, 7 \mathrm{H}, \mathrm{H}-7+\mathrm{H}-2 \mathrm{~b}+\mathrm{H}-19 \mathrm{~b}+$ $\mathrm{H}-22 \mathrm{a}+\mathrm{H}-6), 1.34(s, 3 \mathrm{H}, \mathrm{H}-27), 1.33-1.16$ (m, 4H, $\mathrm{H}-16+\mathrm{H}-1 \mathrm{~b}+\mathrm{H}-21 \mathrm{~b}+\mathrm{H}-22 \mathrm{~b}), 1.15(s, 6 \mathrm{H}, \mathrm{H}-28+$ $\mathrm{H}-25), 1.12$ ( $s, 3 \mathrm{H}, \mathrm{H}-26), 1.09$ ( $s, 3 \mathrm{H}, \mathrm{H}-23), 1.08-$ 0.99 ( $m, 2 \mathrm{H}, \mathrm{H}-5+\mathrm{H}-15 \mathrm{~b}), 0.92(s, 3 \mathrm{H}, \mathrm{H}-24), 0.80$ $(s, 3 \mathrm{H}, \mathrm{H}-29) \mathrm{ppm} ;{ }^{13} \mathrm{C} \mathrm{NMR}\left(125 \mathrm{MHz}, \mathrm{CDCl}_{3}\right): \delta$ $=199.8$ (C-11), 176.9 (C-30), 169.7 (C-13), 128.4 (C-12), 127.0 (C-36), 124.0 (C-37), 83.2 (C-32, $C \equiv \mathrm{C}), 75.8(\mathrm{C}-3), 74.0(\mathrm{C}-33, \mathrm{C} \equiv C), 62.0(\mathrm{C}-9), 55.4$ (C-35), 53.5 (C-5), 51.8 (C-31), 48.4 (C-18), 45.3 (C-14), 44.2 (C-20), 43.2 (C-8), 41.6 (C-34), 41.6 (C-4), 41.3 (C-19), 38.5 (C-1), 37.7 (C-22), 37.1 (C-10), 32.7 (C-7), 32.5 (C-2), 31.8 (C-21), 31.0 (C-17), 28.5 (C-29), 28.3 (C-28), 26.4 (C-15 + C-16), 26.3 (C-23), 23.4 (C-27), 18.6 (C-26), 17.7 (C-6), 17.7 (C-25), 16.7 (C-24) ppm; MS (ESI, $\mathrm{MeOH}): m / z=618.5\left(100 \%,[\mathrm{M}+\mathrm{H}]^{+}\right)$; analysis calculated for $\mathrm{C}_{40} \mathrm{H}_{59} \mathrm{NO}_{4}$ (617.90): C 77.75, H 9.62, N 2.27; found: C 77.63, H 9.74, N 2.12.

Methyl (3ß) 3-[3-(diisopropylamino)-prop-1yn-1-yl] 3-hydroxy-11-oxo-olean-12-en-30-oate hydrochloride 27

Mannich reaction of $24(500 \mathrm{mg}, 1 \mathrm{mmol})$ as described above with diisopropylamine $(0.52 \mathrm{~mL}$, $3.7 \mathrm{mmol})$, formalin $(37 \%, 1.8 \mathrm{~mL}, 14.8 \mathrm{mmol})$ and CuI $\quad(20 \mathrm{mg}, \quad 0.1 \mathrm{mmol})$ followed by chromatographic workup yielded crude 27. A solution of this material in diethyl ether was treated with dry hydrochloric acid at $0{ }^{\circ} \mathrm{C}$. After standing at $4{ }^{\circ} \mathrm{C}$ overnight the precipitate was filtered off and washed with water and diethyl ether to yield 27 (342 $\mathrm{mg}, 55 \%)$ as a colorless solid; $\mathrm{mp} 256-257{ }^{\circ} \mathrm{C}$; $[\alpha]_{\mathrm{D}}$ $=665^{\circ}\left(c=0.52, \mathrm{CHCl}_{3}\right) ;{ }^{1} \mathrm{H}$ NMR $(500 \mathrm{MHz}$, $\left.\mathrm{CD}_{3} \mathrm{OD}\right): \delta=5.62(s, 1 \mathrm{H}, \mathrm{H}-12), 4.24(s, 2 \mathrm{H}, \mathrm{H}-34)$, $3.92(d t, 2 \mathrm{H}, J=6.6,13.3 \mathrm{~Hz}, \mathrm{H}-35), 3.73(s, 3 \mathrm{H}$,
H-31), 2.79 (ddd, 1H, $J=3.3,3.3,10.7 \mathrm{~Hz}, \mathrm{H}-1 \mathrm{a})$, 2.48 ( $s, 1 \mathrm{H}, \mathrm{H}-9), 2.21-2.13$ ( $m, 2 \mathrm{H}, \mathrm{H}-18+\mathrm{H}-2 \mathrm{a})$, $2.06(d d d, 1 \mathrm{H}, J=3.4,13.7,13.7 \mathrm{~Hz}, \mathrm{H}-15 \mathrm{a}), 2.03-$ $1.91(m, 2 \mathrm{H}, \mathrm{H}-16+\mathrm{H}-21 \mathrm{a}), 1.88(d d d, 1 \mathrm{H}, J=2.5$, $4.1,13.5 \mathrm{~Hz}, \mathrm{H}-19 \mathrm{a}), 1.80-1.62$ ( $m, 4 \mathrm{H}, \mathrm{H}-2 \mathrm{~b}+\mathrm{H}-$ $7 \mathrm{a}+\mathrm{H}-6 \mathrm{a}+\mathrm{H}-19 \mathrm{~b}), 1.58-1.50(m, 2 \mathrm{H}, \mathrm{H}-7 \mathrm{~b}+\mathrm{H}-$ $22 \mathrm{a}), 1.51-1.43$ ( $m, 16 \mathrm{H}, \mathrm{H}-36+\mathrm{H}-27+\mathrm{H}-6 \mathrm{~b}), 1.43-$ $1.25(m, 4 \mathrm{H}, \mathrm{H}-21 \mathrm{~b}+\mathrm{H}-1 \mathrm{~b}+\mathrm{H}-22 \mathrm{~b}+\mathrm{H}-16), 1.19$ $1.04(m, 14 \mathrm{H}, \mathrm{H}-25+\mathrm{H}-23+\mathrm{H}-5+\mathrm{H}-24+\mathrm{H}-28+$ $\mathrm{H}-15 \mathrm{~b}), 0.93$ ( $s, 3 \mathrm{H}, \mathrm{H}-26), 0.85$ ( $s, 3 \mathrm{H}, \mathrm{H}-29) \mathrm{ppm}$; ${ }^{13} \mathrm{C}$ NMR $\left(125 \mathrm{MHz}, \mathrm{CDCl}_{3}\right): \delta=200.7(\mathrm{C}-11)$, 177.1 (C-30), 171.3 (C-13), 127.6 (C-12), 93.8 $(\mathrm{C}-32, C \equiv \mathrm{C}), 75.0(\mathrm{C}-3), 74.6(\mathrm{C}-33, \mathrm{C} \equiv C), 61.9$ (C-9), 54.5 (C-35), 53.2 (C-5), $51.0\left(\mathrm{C}-31, \mathrm{OCH}_{3}\right)$, 48.5 (C-18), 45.3 (C-14), 43.9 (C-20), 43.2 (C-8), 41.6 (C-4), 41.0 (C-19), 38.0 (C-1), 37.6 (C-22), 37.0 (C-10), 35.8 (C-34), 32.4 (C-2), 31.6 (C-7), 31.5 (C-17), 30.6 (C-21), 27.7 (C-29), 27.1 (C-28), 26.2 (C-16), 25.9 (C-15), 25.3 (C-24), 22,7 (C-27), 17.8 (C-36), 17.4 (C-6), 17.0 (C-26), 15.9 (C-23), 14.1 (C-25) ppm; MS (ESI, MeOH): $\mathrm{m} / \mathrm{z}=622.6$ (100\%, $\left.\quad[\mathrm{M}-\mathrm{Cl}]^{+}\right)$; analysis calculated for $\mathrm{C}_{40} \mathrm{H}_{64} \mathrm{ClNO}_{4}$ (658.39): C 72.97, H 9.80, N 2.13; found: C 72.69, H 9.96, N 2.00.

Benzyl (3ß) 3-[(3-diisopropylamino)-prop-1yn-1-yl]-3-hydroxy-11-oxo-olean-12-en-30-oate hydrochloride 28

Mannich reaction of 25 (250 $\mathrm{mg}, 0.42 \mathrm{mmol})$ with diisopropylamine ( $70 \mu \mathrm{l}, 0.5 \mathrm{mmol})$, formalin (37\%, $0.2 \mathrm{~mL}, 2.5 \mathrm{mmol})$ and $\mathrm{CuI}(20 \mathrm{mg}, 0.1 \mathrm{mmol})$ as described above, column chromatography $\left(\mathrm{SiO}_{2}\right.$, hexanes/ ethyl acetate $8 / 2 \rightarrow$ methanol) followed by treating the free base with gaseous hydrochloric acid gave 28 (271 $\mathrm{mg}, 63 \%)$ as a colorless solid; mp 253$254{ }^{\circ} \mathrm{C} ;[\alpha]_{\mathrm{D}}=671^{\circ}\left(\mathrm{c} 0.52, \mathrm{CHCl}_{3}\right) ;{ }^{1} \mathrm{H}$ NMR $(500$ $\left.\mathrm{MHz}, \mathrm{CD}_{3} \mathrm{OD}\right): \delta=7.34-7.19(m, 5 \mathrm{H}$, aromat $), 5.36$ $(s, 1 \mathrm{H}, \mathrm{H}-12), 5.14(d, 1 \mathrm{H}, J=12.0 \mathrm{~Hz}, \mathrm{H}-31 \mathrm{a}), 5.00$ $(d, 1 \mathrm{H}, J=12.0 \mathrm{~Hz}, \mathrm{H}-31 \mathrm{~b}), 4.05$ ( $s b r, 2 \mathrm{H}, \mathrm{H}-34)$, 3.80-3.67 ( $m, 2 \mathrm{H}, \mathrm{H}-35), 2.70-2.60$ ( $m, 1 \mathrm{H}, \mathrm{H}-1 \mathrm{a})$, $2.33(s, 1 \mathrm{H}, \mathrm{H}-9), 2.02$ (ddd, $1 \mathrm{H}, J=4.4,13.8,14.0$ Hz, H-15a), 1.98-1.47 (m, 9H, H-6a + H-7 + H-16 + $\mathrm{H}-18+\mathrm{H}-2 \mathrm{a}+\mathrm{H}-21 \mathrm{a}+\mathrm{H}-19), 1.46-1.34(m, 3 \mathrm{H}$, $\mathrm{H}-2 \mathrm{~b}+\mathrm{H}-21 \mathrm{~b}+\mathrm{H}-6 \mathrm{~b}), 1.33-1.27(m, 15 \mathrm{H}, \mathrm{H}-27+$ H-36), 1.27-1.09 ( $m, 4 \mathrm{H}, \mathrm{H}-22+\mathrm{H}-16+\mathrm{H}-1 \mathrm{~b}), 1.10-$ $0.97(m, 13 \mathrm{H}, \mathrm{H}-25+\mathrm{H}-23+\mathrm{H}-5+\mathrm{H}-24+\mathrm{H}-28)$, $0.96-0.88$ ( $m, 1 \mathrm{H} \mathrm{H}-15 \mathrm{~b}), 0.80(s, 3 \mathrm{H}, \mathrm{H}-26), 0.62$ $(s, 3 \mathrm{H}, \mathrm{H}-29) ;{ }^{13} \mathrm{C}$ NMR (125 MHz, $\left.\mathrm{CD}_{3} \mathrm{OD}\right)$ : $\delta=200.7$ (C-11), 176.4 (C-30), 171.1 (C-13), 136.3 $(\mathrm{Ph}), 128.3(\mathrm{Ph}), 128.0(\mathrm{Ph}), 127.6(\mathrm{C}-12), 93.8$ $(\mathrm{C}-32, C \equiv \mathrm{C}), 74.9(\mathrm{C}-3), 74.5(\mathrm{C}-33, \mathrm{C} \equiv C), 66.0$ (C-31), 61.9 (C-9), 53.7 (C-35), 53.1 (C-5), 48.3 (C-18), 45.2 (C-14), 43.8 (C-20), 43.1 (C-8), 41.5 (C-4), 41.0 (C-19), 38.0 (C-1), 37.4 (C-22), 37.0 (C-10), 35.5 (C-34), 32.4 (C-2), 31.6 (C-7), 31.5 (C-17), 30.6 (C-21), 27.6 (C-29), 27.1 (C-28), 26.2 (C-16), 25.9 (C-15), 25.3 (C-24), 22.7 (C-27), 17.8 (C-36), 17.4 (C-6), 17.0 (C-26), 16.5 (C-23), 15.9 (C-25) ppm; MS (ESI, MeOH): $m / z=698.8(100 \%$, $\left.[\mathrm{M}-\mathrm{Cl}]^{+}\right)$; analysis calculated for $\mathrm{C}_{46} \mathrm{H}_{68} \mathrm{ClNO}_{4}$ 
(734.49): C 75.22, H 9.33, N 1.91; found: C 75.04, H 9.51, N 1.81 .

\section{References}

1- R. Csuk, Expert Opin. Ther. Pat. 2014, 24, 913923.

2- R. Csuk, in Novel Apoptotic Regulators in Carcinogenesis, ed. by G. G. Chen and P. B. Lai, Springer, Dordrecht, 2012, pp 267-288.

3- R. H. Cichewicz, S. A. Kouzi, Med. Res. Rev. 2004, 24, 90-114.

4- M. Co, P. Kostelka, P. Eklund-Aakergren, K. Srinivas, J. W. King, P. J. R. Sjoeberg, C. Turner, Green Chem. 2009, 11, 668-674.

5- R. Ferreira, H. Garcia, A. F. Sousa, C. S. R. Freire, A. J. D. Silvestre, W. Kunz, L. P. N. Rebelo, C. S. Pereira, RSC Advances 2013, 3, 21285-21288.

6- J. Yin, H. Ma, Y. Gong, J. Xiao, L. Jiang, Y. Zhan, C. Li, C. Ren, Y. Yang, Am. J. Plant Sci. 2013, 4, 7-15.

7- V. S. Klimakov, A. V. Zorin, S. S. Vershinin, V. V. Zorin, Russian Patent RU2523545C1, 2014; Chem. Abs. 2014, 161201186.

8- F. N. Lugemwa, Molecules 2012, 17, 92749282.

9- M. S. Yunusov, N. G. Komissarova, N. G. Belenkova, Russian Patent RU2270201C1, 2006; Chem. Abs. 2006, 144, 229523.

10- Y.-h. Zhang, T. Yu, Y. Wang, J. For. Res. (Engl. Ed.) 2003, 14, 202-204.

11- A. K. Ressmann, K. Strassl, P. Gaertner, B. Zhao, L. Greiner, K. Bica, Green Chem 2012, 14, 940-944.

12- J. Achrem-Achremowicz, E. Kepczynska, M. Zylewski, Z. Janeczko, Biomed. Chromatogr. 2010, 24, 261-267.

13- R. Csuk, R. Sczepek, B. Siewert, C. Nitsche, Bioorg. Med. Chem. 2013, 21, 425-435.

14- R. Csuk, C. Nitsche, R. Sczepek, S. Schwarz, B. Siewert, Arch. Pharm. 2013, 346, 232-246.

15- A. Barthel, S. Stark, R. Csuk, Tetrahedron 2008, 64, 9225-9229.

16- R. Csuk, K. Schmuck, R. Schäfer, Tetrahedron Lett. 2006, 47, 8769-8770.

17- L. W. Bieber, M. F. da Silva, Tetrahedron Lett. 2004, 45, 8281-8283.

18- L. V. Anikina, D. A. Shemyakina, L. V. Pavlogradskaya, A. N. Nedugov, V. A. Glushkov, Russ. J. Org. Chem. 2014, 50, 11801183.

19- A. I. Govdi, S. F. Vasilevsky, N. V. Sokolova, I. V. Sorokina, T. G. Tolstikova, V. G. Nenajdenko, Mendeleev Commun. 2013, 23, 260-261.
20- R. Csuk, S. Stark, C. Nitsche, A. Barthel, B. Siewert, Eur. J. Med. Chem. 2012, 53, 337-345.

21- M. Arend, B. Westermann, N. Risch, Angew. Chem. Int. Ed. 1998, 37, 1045-1070.

22- L. Pohjala, S. Alakurtti, T. Ahola, J. YliKauhaluoma, P. Tammela, J. Nat. Prod. 2009, 72, 1917-1926.

23- R. Csuk, S. Schwarz, R. Kluge, D. Ströhl, Eur. J. Med. Chem. 2010, 45, 5718-5723.

24- S. Schwarz, R. Csuk, Bioorg. Med. Chem. 2010, 18, 7458-7474.

25- R. Csuk, S. Schwarz, B. Siewert, R. Kluge, D. Ströhl, Z. Naturforsch., B: J. Chem. Sci. 2011, 66, 521-532.

26- G. A. Tolstikov, M. I. Goryaev, L. V. Tolstikova, Dokl. Akad. Nauk SSSR 1967, 173, 1110-1112.

27- L. Huang, X. Yang, Q. Li, H. Luo, X. Hao, Chinese Patent CN103923158A, 2014; Chem. Abs. 2014, 161, 290984.

28- C.-F. Hung, United States Patent US20120129929A1, 2012; Chem. Abs. 2012, 157, 2366.

29- C.-N. Lin, K.-W.Lin, A. M. Huang, T.-C.Hour, S.-C.Yang, Y.-S.Pu, J.-G.Chang, United States Patent US20130109752A1, 2013; Chem. Abs. 2013, 158, 627265.

30- K.-W Lin, A. M. Huang, T.-C. Hour, S.-C. Yang, Y.-S.Pu, C.-N. Lin, Bioorg. Med. Chem. 2011, 19, 4274-4285.

31- C. Gao, F.-J. Dai, H.-W. Cui, S.-H. Peng, Y.He, X. Wang, Z.-F. Yi, W.-W. Qiu, Chem. Biol. Drug Des. 2014, 84, 223-233.

32- Y.-q. Meng, J.-q. Ding, Y. Liu, H.-h. Nie, S. Guan, C. Zou, N. Zhao, H. Chen, B. Cao, Chem. Res. Chin. Univ. 2012, 28, 214-219.

33- Y. Gao, X. Guo, X. Li, D. Liu, D. Song, Y. Xu, Y.; Sun, M.; Jing, Y.; Zhao, L. Molecules 2010, 15, 4439-4449.

34- P. Skehan, R. Storeng, D. Scudiero, A. Monks, J. Mcmahon, D. Vistica, J. T. Warren, H. Bokesch, S. Kenney, M. R. Boyd, J. Natl. Cancer Inst. 1990, 82, 1107-1112.

35- B. Siewert, J. Wiemann, A. Köwitsch, R. Csuk, Eur. J. Med. Chem. 2014, 72, 84-101.

36- B. Siewert, E. Pianowski, A. Obernauer, R. Csuk, Bioorg. Med. Chem. 2014, 22, 594-615.

37- B. Siewert, E. Pianowski, R. Csuk, Eur. J. Med. Chem. 2013, 70, 259-272.

38- B. Siewert, R. Csuk, Eur. J. Med. Chem. 2014, 74, 1-6.

39- D. Thibeault, C. Gauthier, J. Legault, J. Bouchard, P. Dufour, A. Pichette, A. Bioorg. Med. Chem. 2007, 15, 6144-6157. 